Article

\title{
Joint 3D-Wind Retrievals with Stereoscopic Views from MODIS and GOES
}

\author{
James L. Carr ${ }^{1, *}$, Dong L. Wu ${ }^{2}$, Robert E. Wolfe ${ }^{2}$, Houria Madani ${ }^{1}$, Guoqing (Gary) Lin ${ }^{2,3}$ \\ and Bin Tan ${ }^{2,3}$ \\ 1 Carr Astronautics, 6404 Ivy Lane, Suite 333, Greenbelt, MD 20770, USA \\ 2 NASA Goddard Space Flight Center, Greenbelt, MD 20770, USA \\ 3 Science Systems and Applications, Inc., 10210 Greenbelt Road, Suite 600, Lanham, MD 20706, USA \\ * Correspondence: jcarr@carrastro.com; Tel.: +1-301-220-7350
}

Received: 31 July 2019; Accepted: 3 September 2019; Published: 9 September 2019

\begin{abstract}
Atmospheric motion vectors (AMVs), derived by tracking patterns, represent the winds in a layer characteristic of the pattern. AMV height (or pressure), important for applications in atmospheric research and operational meteorology, is usually assigned using observed IR brightness temperatures with a modeled atmosphere and can be inaccurate. Stereoscopic tracking provides a direct geometric height measurement of the pattern that an AMV represents. We extend our previous work with multi-angle imaging spectro-radiometer (MISR) and GOES to moderate resolution imaging spectroradiometer (MODIS) and the GOES-R series advanced baseline imager (ABI). MISR is a unique satellite instrument for stereoscopy with nine angular views along track, but its images have a narrow $(380 \mathrm{~km})$ swath and no thermal IR channels. MODIS provides a much wider $(2330 \mathrm{~km})$ swath and eight thermal IR channels that pair well with all but two ABI channels, offering a rich set of potential applications. Given the similarities between MODIS and VIIRS, our methods should also yield similar performance with VIIRS. Our methods, as enabled by advanced sensors like MODIS and $\mathrm{ABI}$, require high-accuracy geographic registration in both systems but no synchronization of observations. AMVs are retrieved jointly with their heights from the disparities between triplets of ABI scenes and the paired MODIS granule. We validate our retrievals against MISR-GOES retrievals, operational GOES wind products, and by tracking clear-sky terrain. We demonstrate that the 3D-wind algorithm can produce high-quality AMV and height measurements for applications from the planetary boundary layer (PBL) to the upper troposphere, including cold-air outbreaks, wildfire smoke plumes, and hurricanes.
\end{abstract}

Keywords: 3D-winds; atmospheric motion vectors (AMVs); MODIS; GOES-R; ABI; planetary boundary layer (PBL); stereo imaging; parallax

\section{Introduction}

Height assignment using infrared (IR) methods is arguably the largest source of uncertainty in atmospheric motion vectors (AMVs) derived by tracking cloud and water vapor features in satellite imagery. Despite their wide coverage and good sensitivity to motion, the AMV height assignment error (i.e., assigning winds to a wrong height) has been a major obstacle for AMVs to realize their full potential in data assimilation, weather prediction, and atmospheric process studies.

To infer the height of a feature pattern, a conventional AMV height assignment relies on collocated thermal IR radiances in the same images and requires knowledge of an a priori atmospheric thermodynamic profile (e.g., temperature lapse rate) [1]. Such IR-based height assignment methods have at least three fundamental limitations. First, when AMVs are derived from visible imagery and heights are assigned using IR imagery, each spectral band may have different sensitivities to 
cloud layers. This cross-spectral-band height assignment becomes problematic particularly in the case where semi-transparent multi-layer clouds are present [2]. Second, The IR method would fail in an atmosphere that has a small or reversed vertical temperature gradient. The reversed temperature lapse rate can occur in the atmospheric planetary boundary layer (PBL) for low clouds [3] or near the tropopause [4]. Third, the height that characterizes an AMV feature is not necessarily that of the brightest or coldest pixel. Rather, it is determined by a collection of dark and bright (cold and warm) pixels, and the contrast of these pixels relative to the background. These limitations have led to several modifications in IR-based algorithms in which one works better than another depending on the type of cloud scene [5].

The IR-based height assignment is perhaps the best solution if only a single platform is available from space. Simultaneous or near-simultaneous observations from multiple platforms have been rare with sufficiently high-quality geo-registration accuracy. Recently, advanced sensors on low-Earth orbit (LEO) and geostationary Earth orbit (GEO) satellites have achieved superb pointing accuracy to produce high quality imagery with fine spatial resolution. For example, the image navigation and registration (INR) of the GOES-R series advanced baseline imager (ABI) has achieved geo-registration accuracy of a few tenths of a pixel [6,7]. In addition, the moderate resolution imaging spectroradiometer (MODIS) from LEO produces imagery with a similar geolocation accuracy [8]. These advances in GEO and LEO navigation and geo-registration now provide a new life to stereoscopic remote sensing of AMVs from multiple spaceborne platforms.

We shall use the term "3D-winds" in this paper to generically describe our stereo methods since a wind vector is solved for simultaneously with its three-dimensional (3D) position within the air column. This is not to be confused with the sense of the term in wind profiling, which resolves the motion of air parcels at multiple heights along a single column of atmosphere. Stereo methods enable a single wind to be retrieved along with its 3D position for each sight line but has the advantage of being able to cover large swaths using a single pair of platforms. Our previous study has shown that joint AMV retrievals using stereo observations from two platforms can significantly improve the quality of AMV wind and height measurements [9]. This study featured the multi-angle imaging capability of the multi-angle imaging spectro-radiometer (MISR), which can provide stereo-height determinations from a single platform. Although MISR's nine angular views can independently determine the height and the cross-track component of wind, the along-track wind and height are coupled and difficult to separate accurately. By adding GOES-16 ABI imagery, we demonstrated that the joint AMV retrievals uncouple the along-track wind and height to enable improved wind and height products.

The 3D-wind algorithm developed for MISR-GOES is applicable in general to GEO-GEO, LEO-GEO, and LEO-LEO combinations. It requires no synchronization between images acquired by two different platforms. Because of the rapid refresh rate provided by a sensor in GEO (for ABI typically: $10 \mathrm{~min}$. Full Disk, $5 \mathrm{~min}$. CONUS, $1 \mathrm{~min}$. MESO), AMV wind velocity is highly constrained by the GEO image sequence. A snapshot from LEO will provide an additional key constraint on AMV height. The 3D-wind parallax solver takes into account wind-induced apparent parallaxes even if the LEO image is not synchronized with the GEO images. This flexibility allows a single GEO system to pair with neighboring GEOs and many LEOs that fly under its coverage, greatly enhancing the value of each individual platform.

In this paper, we extend the technique demonstrated with MISR and GOES to MODIS and GOES ABI. The extension provides several benefits to global AMV observations with jointly retrieved stereo heights. First, using MODIS will produce a much wider swath relative to MISR for wind and height measurements. The improved coverage provides for more frequent revisits to targets of interest, such as hurricanes and wildfires. Second, MODIS and ABI share several thermal-IR channels, including a water-vapor channel, which allows stereo measurements in daytime and nighttime. Nighttime stereo techniques for AMV winds and heights is relatively new and requires validation from other independent observations. Third, the extended LEO-GEO algorithm will provide the stereo retrievals of AMV winds and heights in the regions where the GEO-GEO stereo is not available because of a 
lack of overlapping coverage. With the successful demonstration of MODIS-GOES 3D-winds, it is not difficult to imagine that the technique can be successful with other LEO instruments such as the visible infrared imaging radiometer suite (VIIRS).

Our materials and methods are fully described in Section 2 of this paper. Section 3 presents our results and discussion. It includes validation and several application cases where vertical resolution is critical to the study of atmospheric dynamics, including the PBL structure during a cold air outbreak, vertically resolved atmospheric dynamics during Hurricane Michael with deep convention, and wildfire smoke plumes. The wildfire case shows the flexibility of stereo methods to study advection of aerosols as well as clouds. These application cases demonstrate the value of using MODIS and GOES together to jointly retrieve AMVs and stereo heights. MODIS-GOES 3D-winds capability will be a useful new tool for atmospheric research to complement existing AMV methods with conventional IR height assignments.

\section{Materials and Methods}

We have implemented our methods in MATLAB, first for MISR and GOES ABI and now for MODIS and ABI as is fully described in Section 2.1 below. Accurate joint AMV and stereo-height retrievals are enabled by the highly accurate geolocation and geo-registration of the MODIS and ABI products, which are our materials, as is explained in Section 2.2.

\subsection{Stereo 3D-Winds Method Using MODIS and GOES}

MODIS spectral channels pair well with those of ABI in accordance with Table 1. Only ABI bands 8 and 13 are not well paired with MODIS. In principle, any of these pairings can be used for joint MODIS-GOES AMV-height retrievals.

Table 1. Moderate resolution imaging spectroradiometer (MODIS) offers many close spectral matches with the advanced baseline imager (ABI) channels.

\begin{tabular}{cccccc}
\hline ABI Band & $\begin{array}{c}\text { Wavelength } \\
(\boldsymbol{\mu m})\end{array}$ & $\begin{array}{c}\text { Spatial } \\
\text { Resolution }(\mathbf{m})\end{array}$ & MODIS Band & $\begin{array}{c}\text { Wavelength } \\
(\boldsymbol{\mu m})\end{array}$ & $\begin{array}{c}\text { Spatial } \\
\text { Resolution }(\mathbf{m})\end{array}$ \\
\hline 1 & $0.45-0.49$ & 1000 & 3 & $0.459-0.479$ & 500 \\
\hline 2 & $0.59-0.69$ & 500 & 1 & $0.620-0.670$ & 250 \\
\hline 3 & $0.846-0.885$ & 1000 & 2 & $0.841-0.876$ & 250 \\
\hline 4 & $1.371-1.386$ & 1000 & 26 & $1.360-1.390$ & 1000 \\
\hline 5 & $1.58-1.64$ & 1000 & 6 & $1.628-1.652$ & 500 \\
\hline 6 & $2.225-2.275$ & 2000 & 7 & $2.105-2.155$ & 500 \\
\hline 7 & $3.80-4.0$ & 2000 & 21,22 & $3.929-3.989$ & 1000 \\
\hline 8 & $5.77-6.6$ & 2000 & - & - & - \\
\hline 9 & $6.75-7.15$ & 2000 & 27 & $6.535-6.895$ & 1000 \\
\hline 10 & $7.24-7.44$ & 2000 & 28 & $7.175-7.475$ & 1000 \\
\hline 11 & $8.3-8.7$ & 2000 & 29 & $8.4-8.7$ & 1000 \\
\hline 12 & $9.42-9.8$ & 2000 & 30 & $9.58-9.88$ & 1000 \\
\hline 13 & $10.1-10.6$ & 2000 & - & & - \\
\hline 14 & $10.8-11.6$ & 2000 & 31 & $10.780-11.280$ & 1000 \\
\hline 15 & $11.8-12.8$ & 2000 & 32 & $11.770-12.270$ & 1000 \\
\hline 16 & $13.0-13.6$ & 2000 & 33 & $13.185-13.485$ & 1000 \\
\hline & & & & &
\end{tabular}




\subsubsection{Process Flow}

The MODIS-GOES 3D-winds process flow is shown in Figure 1. The first step is to bring the imagery from both satellites into a common frame of reference. We have chosen the fixed grid of the GOES-R series ABI into which individual MODIS granules will be remapped. The ABI fixed grid is an idealized representation of the Earth-satellite imaging geometry from geostationary orbit for which all pixels are constructed at standard fixed-grid sites for each GOES satellite reference longitude [10]. GOES ABI product imagery is registered to the ellipsoid (effectively WGS 84) so that the line-of-sight to each pixel is traced from an idealized satellite along that line-of-sight to a pierce-point on the ellipsoid. On the other hand, MODIS L1b products are navigated to the terrain surface and are not resampled to standard fixed sites [11].

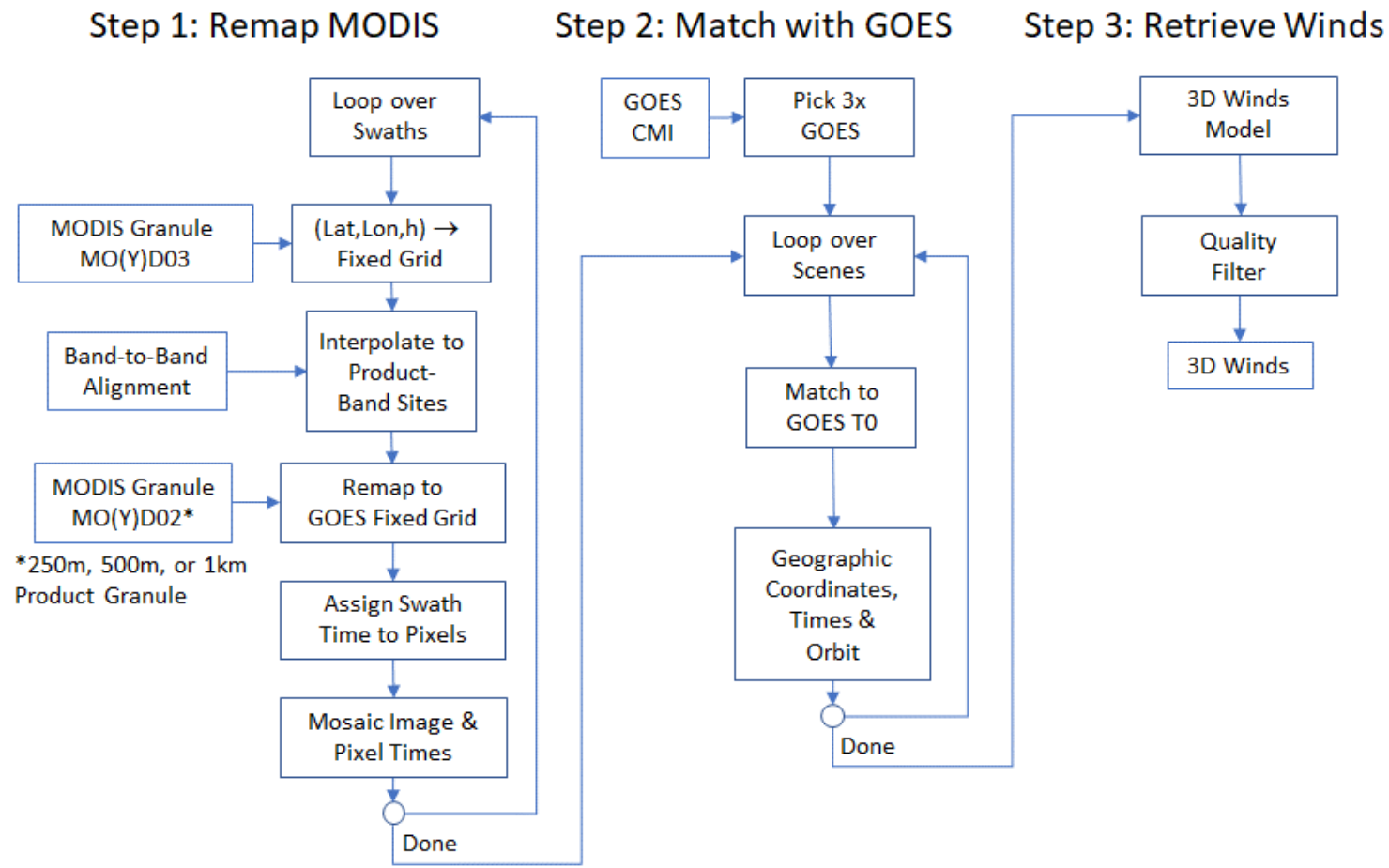

Figure 1. Creation of a 3D-winds dataset is a three-step process. Step 1 remaps a moderate resolution imaging spectroradiometer (MODIS) L1b granule into the fixed-grid projection of the advanced baseline imager (ABI). Step 2 matches features common to a triplet of GOES cloud and moisture imagery (CMI) or radiances and the remapped MODIS granule to measure disparities between apparent geographical positions, which are affected by both atmospheric motion and parallax. Step 3 interprets the observed disparities as atmospheric motion vectors (AMVs) with vertical height assignments.

In this paper, we use the nomenclature "MOD" to indicate MODIS on Terra and "MYD" to indicate MODIS on Aqua. MODIS granules contain 5 minutes of imagery labeled by the year, day of year, hour, minute, and collection; e.g., MOD2018196.1700.061 is Terra, day 196 of 2018 (July 15th), beginning at 17:00, from Collection 6.1. This nomenclature is stylistically similar to the MODIS granule file names. The MODIS geolocation data granules ("MOD03" files for the Terra satellite and "MYD03" files for the Aqua satellite) provide the geographic coordinates for MODIS pixels at a nominal $1 \mathrm{~km}$ nadir resolution. These coordinates are triplets of geodetic latitude, longitude, and terrain height above mean sea level (i.e., the geoid). In the case of a clear-sky pixel, these coordinates would truly represent the geodetic location of the pixel; if an atmospheric feature, such as a cloud, they would instead represent the point at which the line-of-sight from MODIS through the pixel's assigned coordinates pierces the terrain surface. The difference would depend on the off-nadir scan angle and the height of the feature above the terrain. To work in the same frame of reference as ABI, we extend the line-of-sight from MODIS 
to the WGS-84 ellipsoid and recalculate the geographic coordinates to represent the pierce-point on the ellipsoid, as illustrated in Figure 2. Each MODIS scan is remapped on its own and placed into a fixed-grid mosaic. In general, there is overlap between the coverage of successive swaths as the swath height grows away from nadir ("bowtie" effect) and there can also be gaps in the coverage near nadir. Only the first duplicated pixel is retained in the mosaic and the coverage gaps are filled using spatial interpolation.

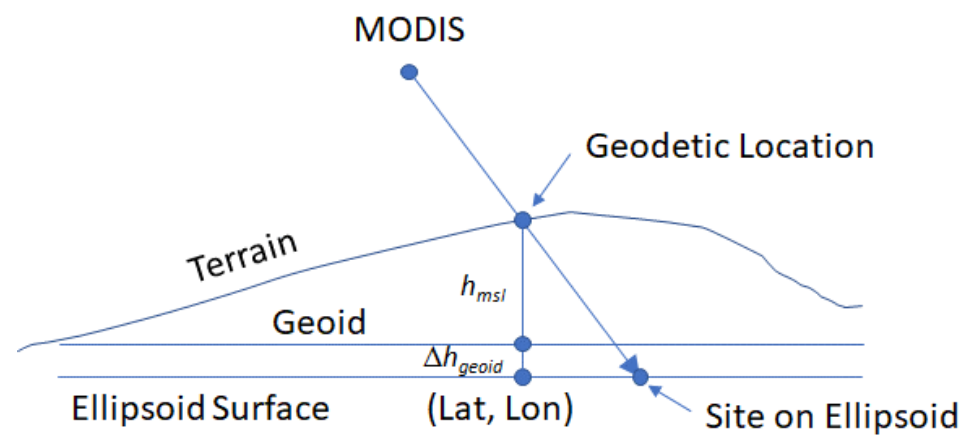

Figure 2. MODIS pixel geographic coordinates represent the geodetic location where the line-of-sight pierces the terrain surface. We recalculate the geographic coordinates to represent where the line-of-sight pierces the WGS-84 ellipsoid considering the terrain height above the geoid (i.e., mean sea level) and the height of the geoid above the ellipsoid (gravity model).

Accurate time tagging of pixels is essential for the 3D-winds application. The center time of each MODIS swath is recorded in atomic time relative to the universal-time epoch of midnight 1 January 1993 (TAI93). We use swath-center times and knowledge of the MODIS scan rate to assign a time tag to each pixel before it is remapped. These time tags are remapped along with the radiances to provide time tags for the MODIS mosaic in the ABI fixed grid. It is a little more difficult to estimate the time tags for the ABI pixels. The ABI scans the Earth in 22 swaths for a full-disk (FD) scene, six swaths for a continental U.S. (CONUS) scene, and two swaths for a mesoscale (MESO) scene of $1000 \mathrm{~km} \times 1000 \mathrm{~km}$ (nominal sub-satellite point distances). The scan pattern is defined in a "timeline" for instrument activities that is commanded to the satellite. The timeline will determine the times for pixels up to an uncertainty near the stitching line between swaths. We accept this ambiguity as unavoidable and model the times according to the swath to which each pixel is most likely to belong and the timeline definition. Quality screening later in the processing will eliminate those few cases where the time tag has been incorrectly assigned. Figure 3 shows an ABI CONUS scene, a MODIS granule remapped into the ABI fixed grid, and their respective modeled time tags. The six swaths of the ABI CONUS scene are clearly seen. With the mode 3 and 6 timelines, each swath follows its predecessor by 30 seconds and time advances $\sim 6$ seconds from the west to the east. MODIS times advance along the ground track and are also organized in swaths, but they are only $\sim 10 \mathrm{~km}$ tall, much smaller than the ABI swaths, and therefore not readily apparent. MODIS times also advance west to east on the descending pass, but only by $\sim 1$ second. 
(a)

GOES-16 CONUS Scene 2018/196 17:02:26.6

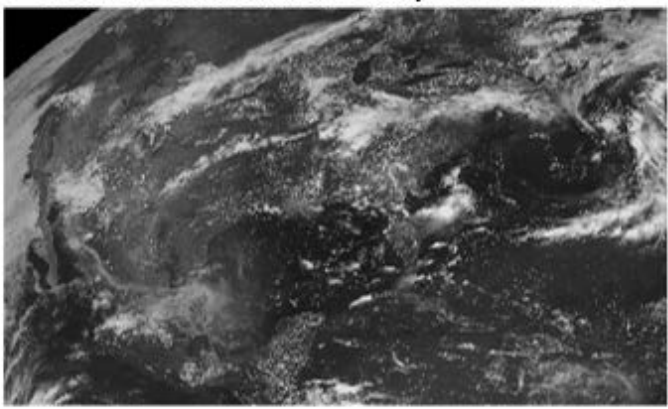

GOES Pixel Times (s)

(c)



mapped MODIS Granule MOD20181961700.061

(b)

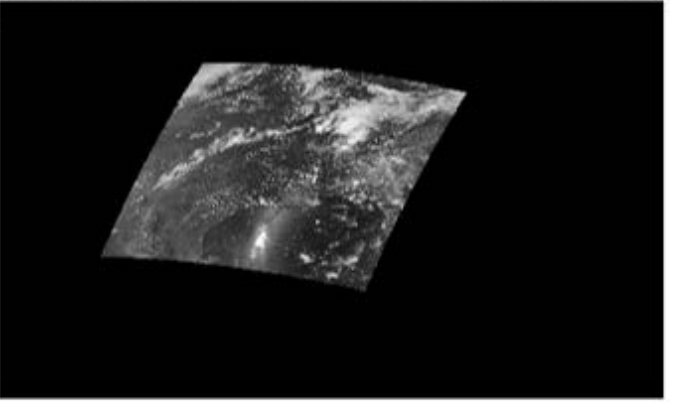

MODIS Pixel Times (s)

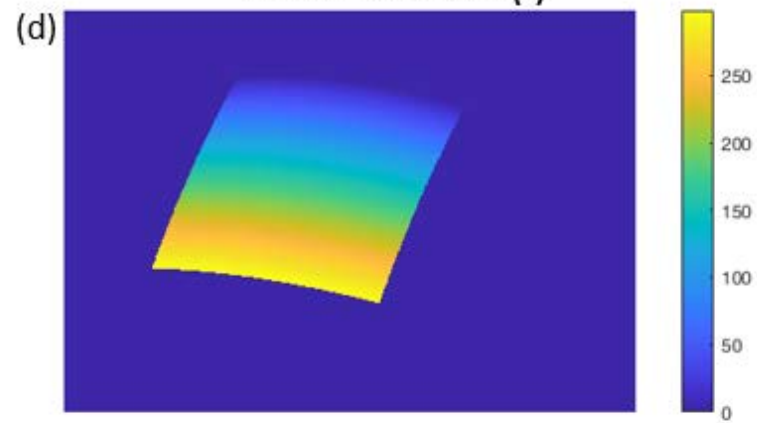

Figure 3. MODIS radiances are remapped along with their time tags to form fixed-grid mosaics in the same geometry as GOES ABI. An ABI scan model provides the time tags for the ABI pixels in each scene. At the upper left (a) is the B02 GOES-16 cloud and moisture imagery (CMI) for the CONUS scene and at the upper right $(\mathbf{b})$ is the matching MODIS granule remapped to the ABI fixed grid. Their respective time models are shown on the bottom row (c,d). A common time epoch is used, which is the time of the first center-of-scan in the MODIS granule (about one second after the granule's product time in this case). The case shown is MODIS granule MOD2018196.1700.061 (MODIS Band 1) paired with GOES-16 CONUS ABI Band 2.

The ABI repetitively follows its commanded timeline and will usually acquire a FD scene every 10 minutes, a CONUS scene every five minutes, and either one or two distinct MESO scenes every 30 seconds (if one MESO) or 60 seconds (if two distinct MESOs). We work with triplets of a given scene type and select the middle scene (time: $T_{0}$ ) so that it is most simultaneous with the MODIS granule being processed. Our second step matches templates taken from the GOES ABI scene $T_{0}$ to locate the feature represented in each template in the other two ABI scenes $\left(T_{0}-\Delta \mathrm{t}\right.$ and $T_{0}+\Delta \mathrm{t}$, where $\Delta \mathrm{t}$ is the refresh time between timelines) and matches the template against the MODIS mosaic as well. The apparent shift of the template relative to its nominal location is determined to subpixel accuracy through normalized cross correlation (NCC) with interpolation on the NCC correlation surface [12]. These shifts are the disparities (illustrated in Figure 4) that represent a combination of motion and parallax between the two satellite vantage points. We also perform an autocorrelation of the template with the $\mathrm{ABI}$ scene $T_{0}$, from where the template is drawn, to eliminate templates that are too spatially uniform to provide adequate feature matches. 


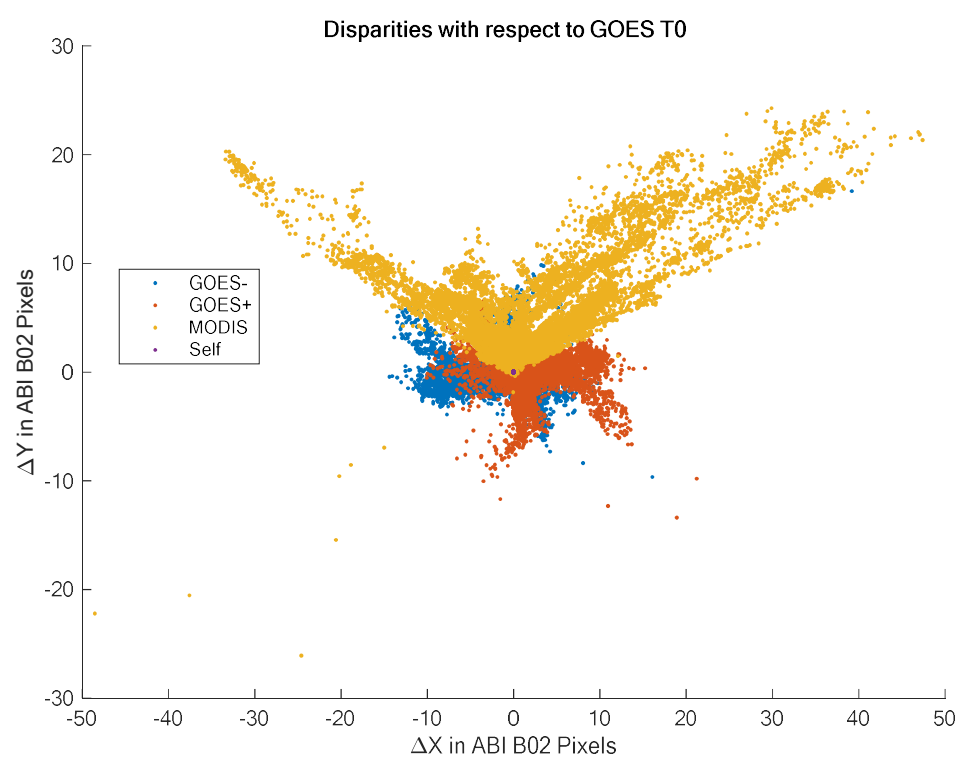

Figure 4. Disparities between matched features in GOES ABI scene $T_{0}$ and the other images measure a combination of motion and parallax. Disparities are measured along the fixed grid axes $(+X-$ east, $+Y-$ north) in units of ABI B02 pixels. The legend identifies the match from GOES ABI $T_{0}$ to $T_{0}-\Delta \mathrm{t}$ ("GOES-"), $T_{0}+\Delta \mathrm{t}$ ("GOES+"), MODIS, and to itself ("Self"). The characteristic wing pattern seen in the MODIS disparities with respect to the central image of the GOES triplet is due to the large MODIS scan angle at either end of its swath. The self matches (or autocorrelations) are tightly clustered at the origin. The case shown is MODIS granule MOD2018196.1700.061 (MODIS Band 1) paired with GOES-16 CONUS ABI Band 2.

The last step is the interpretation of the disparities as 3D-winds model states. Each template site is assigned a three-dimensional vector $\vec{\delta}=\vec{\delta}_{0}+\vec{v} \cdot\left(t-t_{0}\right)$, represented in Figure 5 , to lift it off the template's assigned place on the WGS-84 ellipsoid as a function of time relative to the assigned time $\operatorname{tag} t_{0}$ for the template. The initial vector displacement $\vec{\delta}_{0}$ plus the two components of the horizontal wind field $\vec{v}$ are the five model states per site. The model requires satellite position vectors for Terra or Aqua and the GOES satellite. The former is included in the MODIS geolocation data granule from which we interpolate to the time assigned to the template and the latter is taken from a GOES satellite mission-life ephemeris (courtesy of Marco A. Concha and the NASA GOES-R Program). The model used is identical to the MISR-GOES 3D-winds model without bundle adjustment that is fully derived in the Appendix of Carr, Wu, Kelly, and Gong [9]. It can also be used for GEO-GEO and LEO-LEO 3D-winds and is therefore a generic multi-satellite model. 


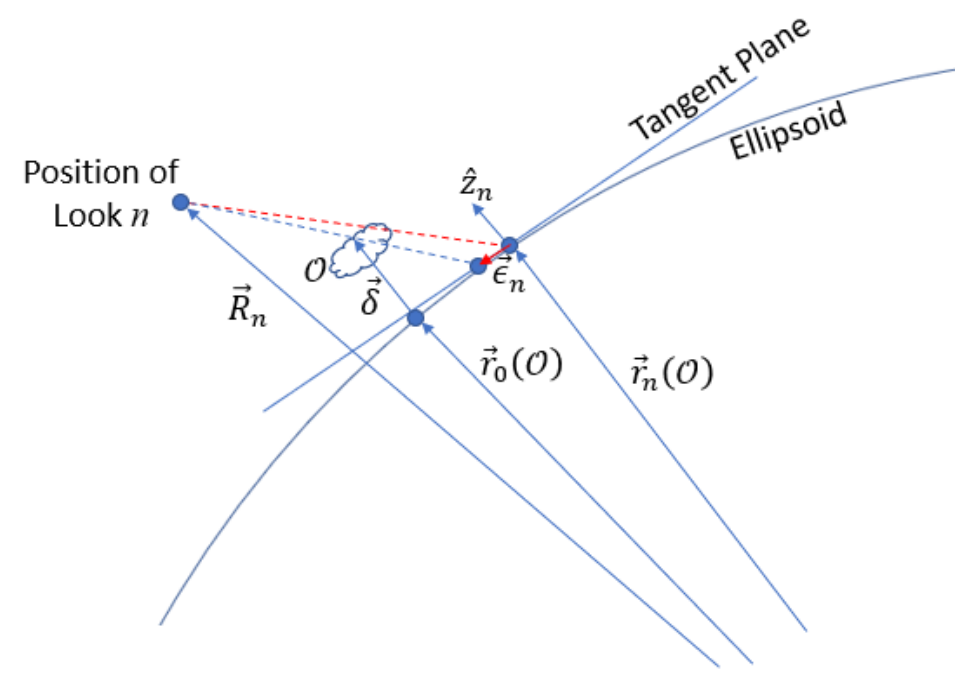

Figure 5. The MISR-GOES 3D-winds retrieval model is reused for MODIS and GOES. The $n=0$ reference image provides a template for the feature $O$ with nominal location $\vec{r}_{0}(O)$ where it projects onto the ellipsoid as seen from camera look $n=0$. The ellipsoid location of the matched feature $\vec{r}_{n}(O)$ in $n \neq 0$ is determined from the measured disparity between $n \neq 0$ and $n=0$. The model minimizes the weighted sum of the squares across all looks of the calculated differences $\vec{\epsilon}_{n}$. Each $\vec{\epsilon}_{n}$ measures the difference in the tangent plane for $n \neq 0$ between where the model places the feature and where it was actually found. The minimization uses five model states for each retrieval and the problem is solved by iterative refinement of the states until converged because the problem is slightly nonlinear. Three position states $\vec{\delta}_{0}$ describe the displacement of the template from its nominal location on the ellipsoid $\vec{r}_{0}(O)$ at the assigned time tag $t_{0}$ for the template. The vertical component of $\vec{\delta}_{0}$ is the height assignment. Two more states describe the motion of the template (wind) so that its instanteous displacement is $\vec{\delta}=\vec{\delta}_{0}+\vec{v} \cdot\left(t-t_{0}\right)$, where $\vec{v}$ is an AMV describing horizontal motion.

The retrieval model will generally leave residuals that are a fraction of one ABI pixel. Figure 6 shows an example where each residual displacement is resolved along the $\mathrm{U}(+$ East) and V (+north) directions in the tangent plane. The U-V notation is adopted for the rest of the paper to designate vector components in the cardinal directions that are either displacements or velocities. Figure 7 shows an example of the retrieved 3D-winds states. Sites for which the model does not explain the disparities are presumed anomalous and are screened out using a statistical plausibility test on the residuals. 

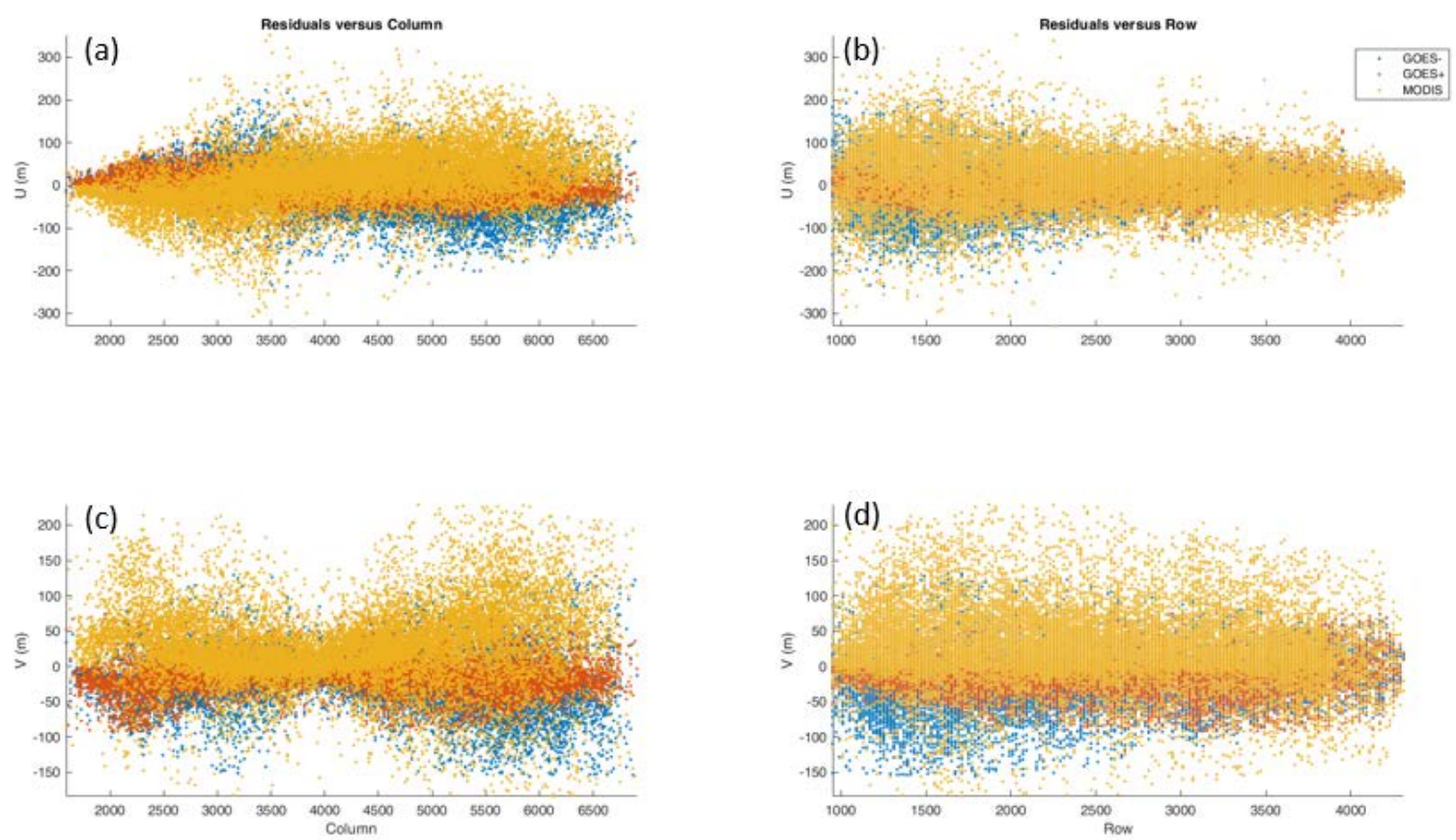

Figure 6. Residual disparities after applying the 3D-winds retrieval model in the tangent plane are plotted by image column in $(\mathbf{a}, \mathbf{c})$ or row in $(\mathbf{b}, \mathbf{d})$. The legend identifies the matches used in the retrievals: i.e., from GOES ABI $T_{0}$ to $T_{0}-\Delta \mathrm{t}$ ("GOES-"), $T_{0}+\Delta \mathrm{t}$ ("GOES+"), and MODIS. Residuals are less than 0.5 ABI B02 pixel, which is $500 \mathrm{~m}$ at the subsatellite point. The case shown is MODIS granule MOD2018196.1700.061 (MODIS Band 1) paired with GOES-16 CONUS ABI Band 2.


Figure 7. Retrieved 3D-winds states describe the horizontal wind velocities and their pattern heights. Vector lengths in the righthand panel (b) are scaled in proportion to wind speed (length scale shows a $2 \mathrm{~m} / \mathrm{s}$ wind speed) and color-coded according to assigned height (color bar shows height scale). The MODIS true-color composite is shown in the left-hand panel (a) for context. Sun glint is apparent in the MODIS composite, which is not found in the GOES imagery because of the difference in viewing geometry between low Earth orbit (LEO) and geostationary Earth orbit (GEO) satellites. The algorithm is unsuccessful in finding matches in the glint region. Any anomalous retrievals (typically $<<1 \%$ ) may be filtered out in a post-processing step or an assimilation quality control test. The case shown is MODIS granule MOD2018196.1700.061 (MODIS band 1) paired with GOES-16 CONUS ABI Band 2. (Note: retrieval sites are thinned 2:1 in each direction for clarity.). 


\subsubsection{Band-to-Band Adjustments}

Using the full range of paired MODIS-ABI channels requires that the MODIS geolocation granule values be corrected for known band-to-band registration (BBR) offsets in the along-scan and along-track (cross-scan) directions. The BBR offsets are defined with respect to MODIS Band 1 and are available for the pre-launch phase and on-orbit operations using the on-board spectro-radiometric calibration assembly (SRCA) [13,14]. The values for year 2017 are provided in Table 2 for Terra and Aqua for the MODIS bands [15] that have a matching GOES ABI Band as shown in Table 1.

Table 2. MODIS band-to-band registration (BBR) offsets in kilometers with respect to MODIS band 1 for 2017.

\begin{tabular}{ccccc}
\hline MODIS Band & $\begin{array}{c}\text { Aqua Along-Scan } \\
\mathbf{( k m )}\end{array}$ & $\begin{array}{c}\text { Aqua Along-Track } \\
\mathbf{( k m )}\end{array}$ & $\begin{array}{c}\text { Terra Along-Scan } \\
\mathbf{( k m )}\end{array}$ & $\begin{array}{c}\text { Terra Along-Track } \\
(\mathbf{k m})\end{array}$ \\
\hline 2 & -0.0033 & -0.0075 & -0.0033 & -0.0083 \\
3 & 0.0279 & -0.0184 & 0.0264 & 0.0226 \\
6 & -0.2084 & 0.2725 & -0.0577 & -0.0562 \\
7 & -0.2007 & 0.4058 & -0.0141 & -0.0409 \\
22 & -0.2317 & 0.2186 & -0.0473 & -0.1131 \\
26 & -0.2043 & 0.3278 & 0.0749 & -0.0643 \\
27 & -0.1766 & 0.2928 & -0.1103 & 0.0038 \\
28 & -0.1946 & 0.3136 & -0.0765 & 0.0163 \\
29 & -0.2034 & 0.3253 & -0.0443 & 0.0267 \\
30 & -0.2310 & 0.3466 & -0.0484 & 0.0506 \\
31 & -0.2467 & 0.1923 & 0.0137 & -0.0406 \\
32 & -0.1805 & 0.2103 & 0.1238 & -0.0425 \\
33 & -0.2770 & 0.3527 & -0.0208 & 0.0432 \\
\hline
\end{tabular}

If the BBR offsets are uncorrected, the retrieval model cannot fit the disparities, leaving the residuals highly structured and indicative of a systematic error (e.g., Figure 8). The structure disappears once the correction is applied (Figure 9) when remapping into the ABI fixed grid. 



Figure 8. Residual disparities in the tangent plane after retrievals without correcting for band-to-band registration (BBR) offsets (versus longitude $(\mathbf{a}, \mathbf{c})$ and latitude $(\mathbf{b}, \mathbf{d})$ ). The case shown is MODIS granule MYD2019057.2000.061 (Band 31) paired with GOES-16 CONUS ABI band 14.
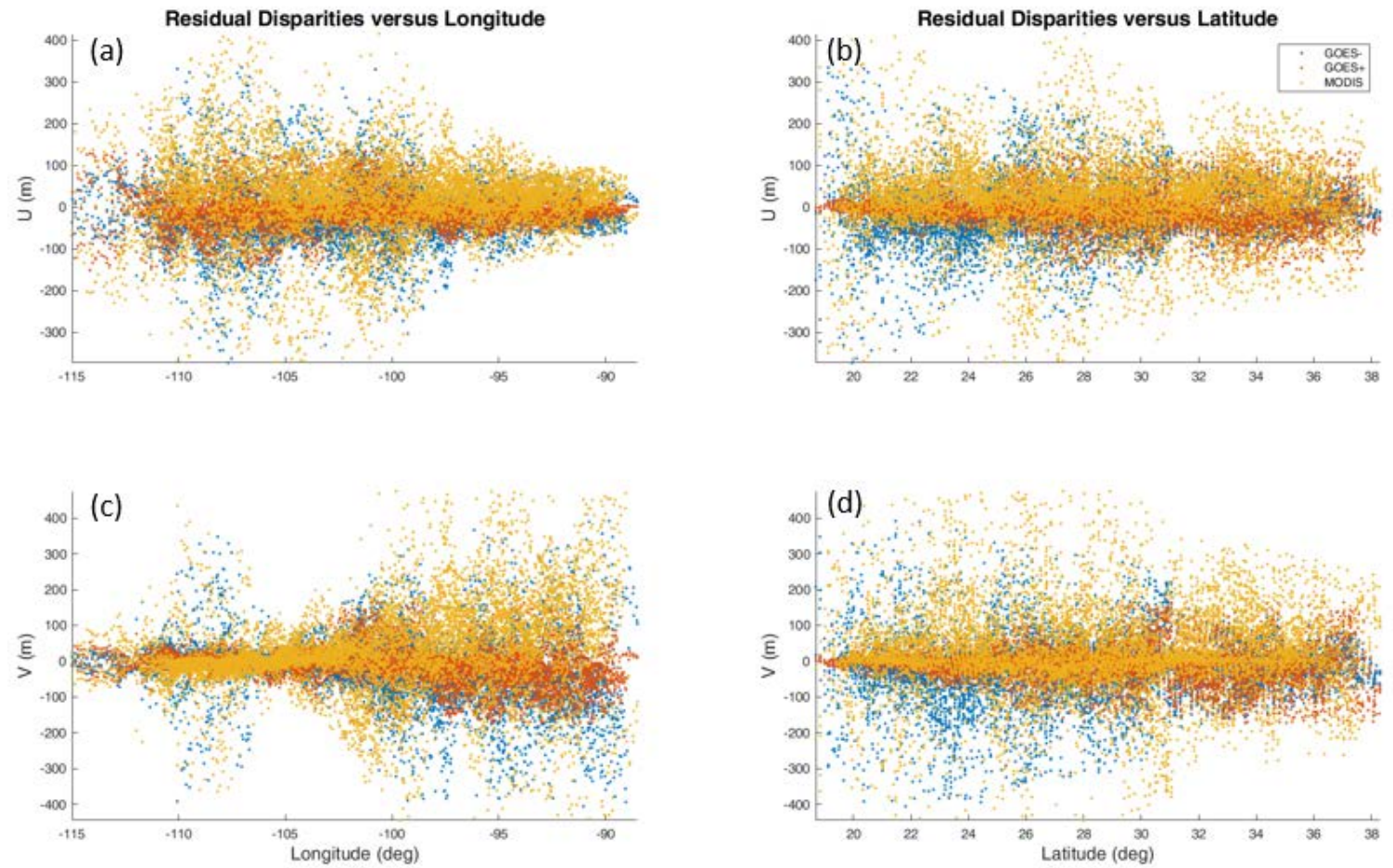

Figure 9. Residual disparities in the tangent plane after retrievals after correcting for the BBR offsets (versus longitude (a,c) and latitude (b,d)). The case shown is MODIS granule MYD2019057.2000.061 (band 31) paired with GOES-16 CONUS ABI band 14. For context, the ground footprint of an ABI band 14 pixel is $2 \mathrm{~km}$ at the subsatellite point 


\subsubsection{Singular Geometry}

The retrieval process provides a means to estimate the uncertainty of the retrieved model parameters through a five-dimensional covariance matrix at each site. This covariance matrix represents the uncertainty statistics for the retrieved parameters (two velocity and three position vector components resolved in a local tangent plane) assuming errors in measuring the disparities are Gaussian and independent. We generally assume that the disparity measurement errors are on the order of one-half the nominal ground sample distance for the ABI channel being used. In the example shown in Figure 7, the MODIS granule is centered at around $92.5^{\circ} \mathrm{W}$ longitude and $35^{\circ} \mathrm{N}$ latitude. This is a favorable geometry. The covariance matrix is symmetric and positive-definite and the square root of each of its eigenvalues describes a principal axis for a one-sigma uncertainty volume. The square roots of the diagonal elements of the covariance matrix describe the one-sigma standard error for each state. Figure 10 shows the state-space volume uncertainty and the standard errors for the retrieved height and wind vector components for this case. The small range of values for the velocity states and the large range for the height is indicative of the fact that the GEO triplet provides most of the information about the wind velocity states and the LEO and GEO sensors working together provide the information about the height. The uncertainty of the height retrievals is quite sensitive to the MODIS scan angle.
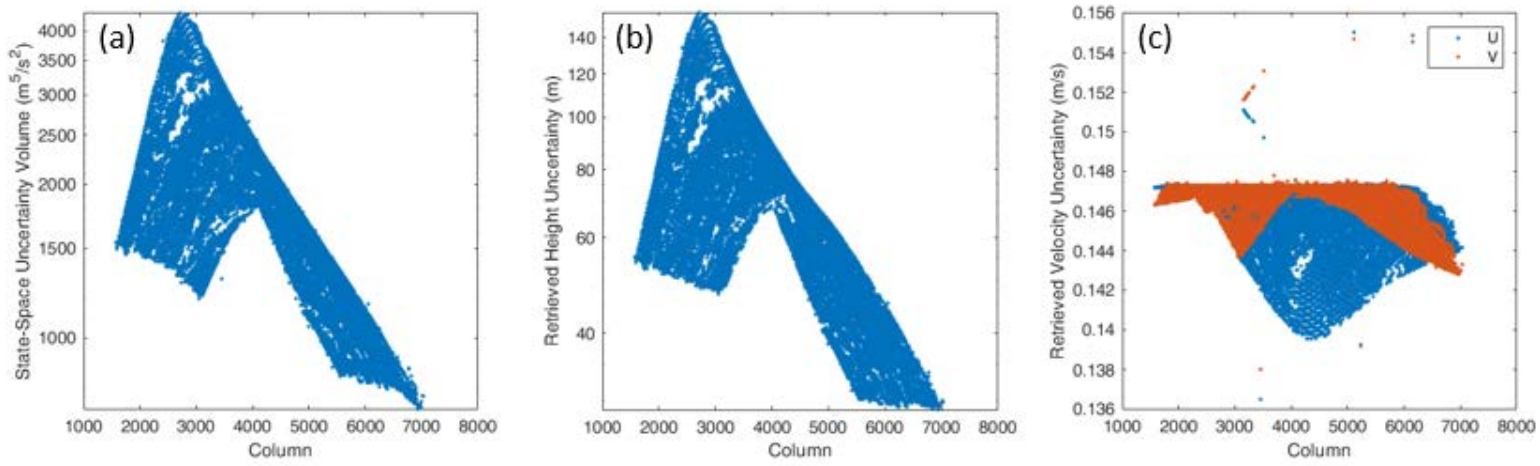

Figure 10. The state-space uncertainty volume (a) and state standard errors $(\mathbf{b}, \mathbf{c})$ are estimated from the covariance matrix from the retrieval performed at each site. The case shown is MODIS granule MOD2018196.1700.061 (MODIS Band 1) paired with GOES-16 CONUS ABI Band 2. Some out-of-family covariances are calculated when the retrieved parallax corrections to site locations place a retrieved site at an inappropriate location. These retrievals are generally filtered out in later quality screening.

Less favorable geometries will exhibit larger error estimates and there exists a singular geometry where the line of sight for MODIS will be exactly parallel with that of ABI and the covariance matrix will have at least one infinite eigenvalue in the singular geometry, expressing the fact that height cannot be retrieved from parallax in this configuration. This will generally occur when MODIS crosses the equator near the subsatellite point for the GOES satellite, which can occur in a Full Disk scene. In the neighborhood of the singular geometry, the uncertainty for the retrieved height will be large and the retrieved height will be subject to large errors. Figure 11 shows the uncertainties when MODIS passes over Ecuador, which is near the GOES-16 subsatellite point. The height uncertainty suffers the most. We can use the standard error estimates to determine the quality of the retrieval and those with standard error on the height assignment greater than $1 \mathrm{~km}$ should not be trusted. Figure 12 shows the geographic distribution of height uncertainties for this case. 

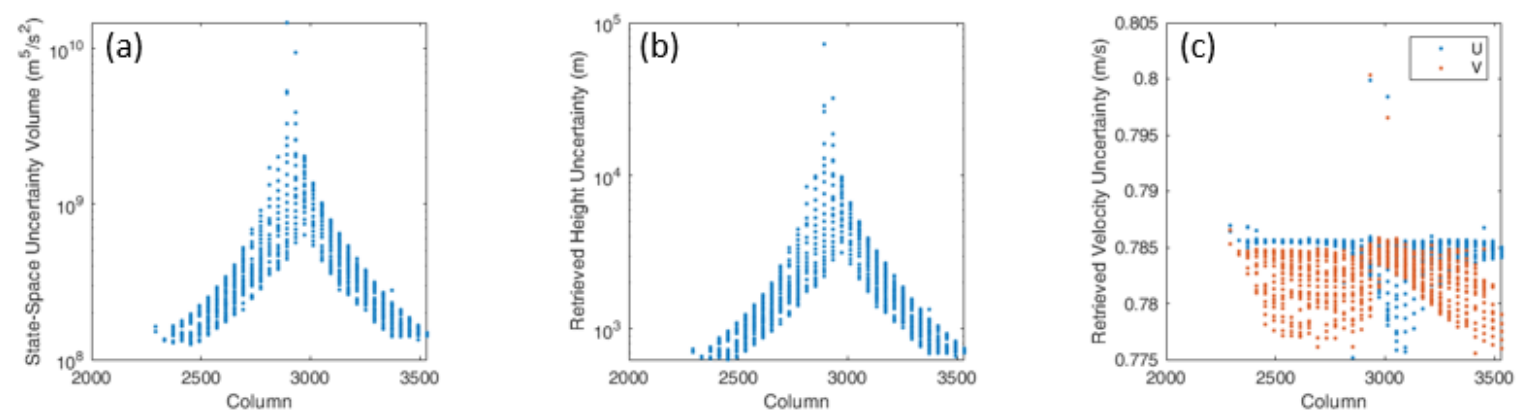

Figure 11. The state-space uncertainty volume (a) and state standard errors $(\mathbf{b}, \mathbf{c})$ depend on the geometry of the LEO satellite with respect to the GEO satellite. The state-space uncertainty volume and standard error for the retrieved height become infinite in the singular geometry. The case shown is MOD2019057.1510.061 (MODIS Band 31) paired with GOES-16 Full Disk Band 14. The height uncertainties are larger than those with MODIS Band 1 paired with ABI Band 2 to begin with due to the larger pixel footprint of the IR channels (four times larger in comparison to the finest resolution) and asymptotically approach infinity as the singular geometry is approached. The longer refresh time for a Full Disk scene compared to a CONUS scene will reduce the velocity uncertainties; but real-world features can change shape over longer periods of time, which is an error not represented here.

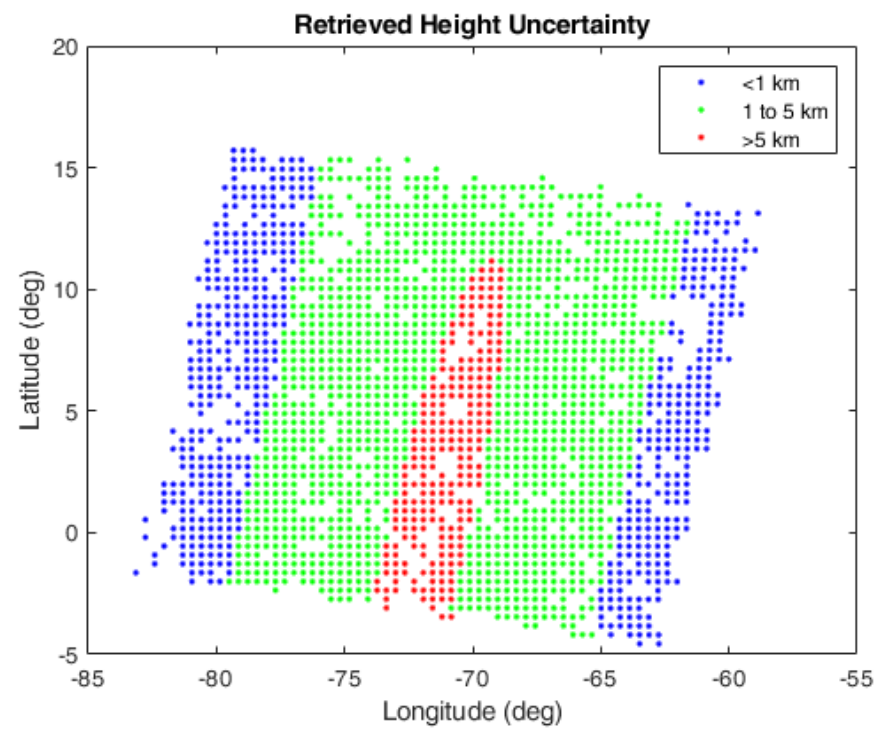

Figure 12. The geographic distribution of retrieved height uncertainty becomes very large when crossing the equator near the GOES subsatellite point, which is where the MODIS scan can be exactly parallel to that of GOES and height becomes unobservable. The case shown is MOD2019057.1510.061 (MODIS Band 31) paired with GOES-16 Full Disk Band 14.

\subsection{Geometric Quality}

Image geometric quality is critical for joint 3D-wind retrievals, both in terms of the accurate assignment of geographic coordinates to pixels ("navigation") and their stability between successive frames of the same scene ("registration") in the case of the GOES-R series ABI. Accurate 3D-wind retrievals are enabled by the outstanding geometric accuracy of observations from both the MODIS and $\mathrm{ABI}$ instruments, which has been achieved as a result of many years of development and investment on the parts of the MODIS and GOES-R programs. 


\subsubsection{MODIS}

MODIS geometric accuracy had been driven by the needs of the terrestrial research community, and a goal since well before the first MODIS was launched on Terra in 1999. During the design and prelaunch testing of the MODIS instruments and the Terra and Aqua spacecraft, including the development of the attitude control systems and the approach to satellite tracking, careful attention was paid to the requirements for position and pointing knowledge accuracy with the goal to minimize any errors that could not be corrected after launch. Before launch, a global set of accurate finer-resolution Landsat-based ground control points were collected to enable early post-launch bias correction, anomaly detection, and removal of any long-term trends. In addition, a detailed global terrain model was developed prelaunch for accurate terrain correction. Careful analysis has been and continues to be performed to monitor and correct for long-term trends in pointing biases and improve the quality of each collection. This includes extrapolation of within-orbit and annual trends for forward processing and deriving the best available trends for reprocessing.

The MODIS instruments on the Terra and Aqua platforms were launched in 1999 and 2002, respectively. Their radiometric $\mathrm{L} 1 \mathrm{~B}$ and geolocation data products have gone through a few rounds of improvements through reprocessing for each new collection. L1 data products are available now in Collection 6.1. Geolocation errors are detected through ground control point matching and time-dependent biases are corrected [16]. Currently, the mission-wise geolocation biases are within 5 meters and root-mean-square errors (RMSEs) are within 55 meters in both the along-track direction and along-scan directions [17]. These geolocation accuracy values are for MODIS Band 1. Figure 13 shows time series of the measured geolocation errors across the entire Collection 6.1 for both satellites. For other bands, there are band-to-band registration offsets as shown in Table 2 in this paper that are applied to achieve similar accuracy. MODIS observations are expected to last into middle of the next decade, or around 2025, before Terra and Aqua are finally retired. 
(a)
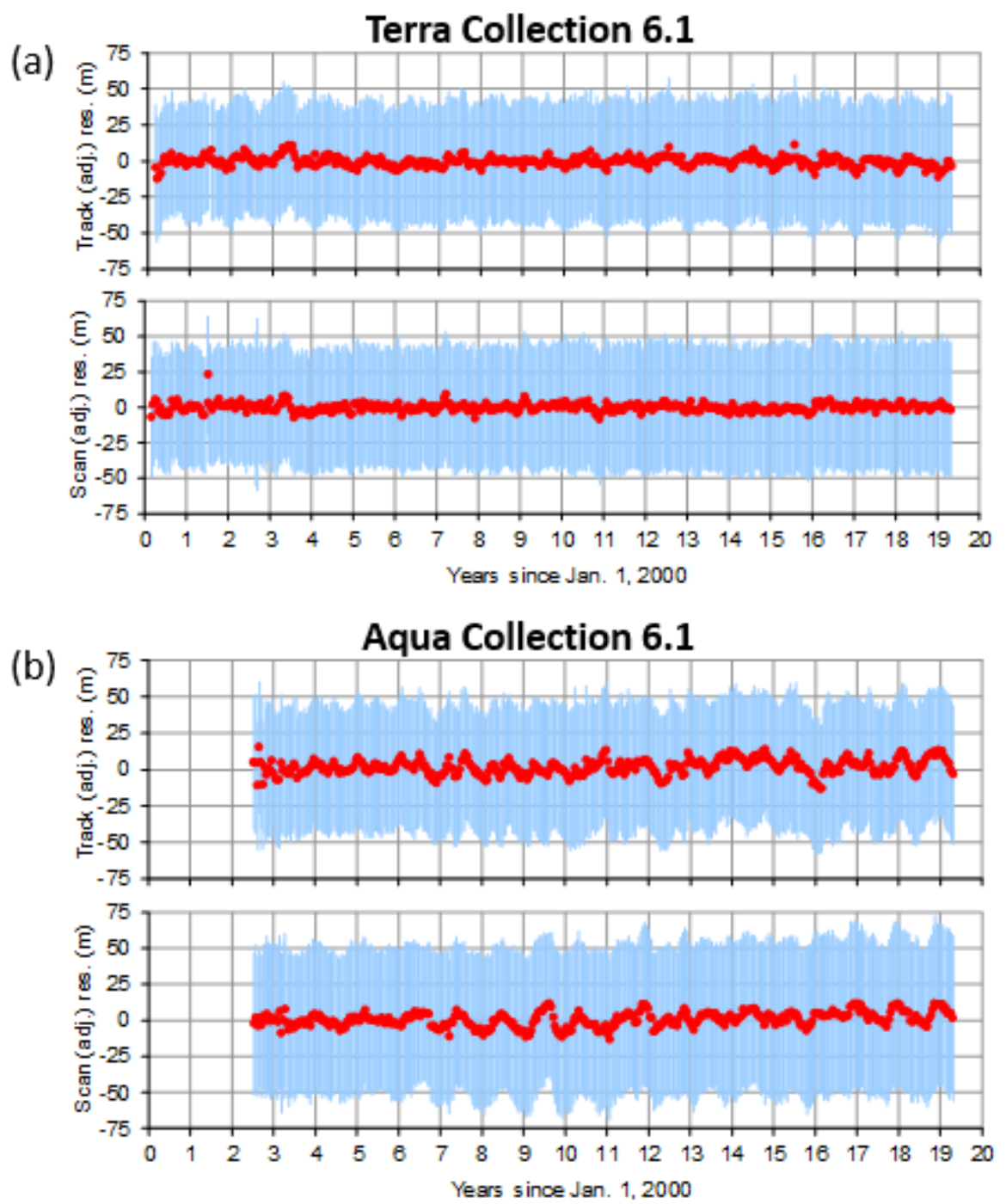

Figure 13. MODIS band 1 measured geolocation error is remarkably stable across all of collection 6.1 for both Terra (a) and Aqua (b). Each point plotted is the sample mean with one-sigma error bars for a 16-day sample of geolocation errors measured using ground control points. The geolocation error has been adjusted to a subsatellite-point equivalent distance and only the daylight portion of the orbit for each satellite is used since MODIS band 1 is a reflective channel.

The VIIRS instrument shares similar spectral bands with MODIS [18] and has similar geolocation accuracy $[19,20]$. VIIRS flies on the Suomi National Polar-orbiting Partnership (S-NPP) and the first Join Polar Satellite System (JPSS-1) platforms that were launched in 2011 and 2017, respectively. Three more copies of VIIRS will be launched onboard JPSS-2/3/4. VIIRS observations are expected to last to 2040, along with those of ABI. Therefore, the usefulness of the 3D-winds retrieval method described in this paper extends several decades into the future.

\subsubsection{GOES}

GOES-R series geometric accuracy has been driven by meteorological requirements, particularly the need for stable imagery for satellite winds applications in which the Earth can be said to stand still and only the clouds move. The INR technology to achieve this objective has been the subject of consistent improvement efforts over the course of the previous GOES series, culminating with the GOES-R series (GOES-16 and -17). GOES is an operational system and unlike MODIS, ABI imagery is not reprocessed into new collections. The INR performance at the present time will solely determine the geometric quality of an image. 
GOES-R series ABI image quality is evaluated with the INR performance assessment tool set (IPATS) [7]. ABI navigation accuracy is assessed by matching ABI imagery with small Landsat 8 template images, called chips, that have been remapped into the ABI fixed-grid projection at a much finer resolution than that of ABI. Table 3 shows the best spectral pairings between the Landsat 8 and GOES ABI channels. The geolocation accuracy of Landsat 8 images is within 15 meters, which is sufficiently accurate to be considered as "true geolocation data" for assessing the geo-registration accuracy (or "navigation") of GOES ABI images. The INR for ABI improved through the post-launch tuning process for GOES-16. The misregistration improved from more than $3 \mathrm{~km}$, at the beginning of the mission, to less than $70 \mathrm{~m}$, after the latest major INR tuning in April 2018 (Figure 14). It should be noted that the linear distance is nadir-equivalent distance converted from the angular space of the fixed-grid projection.

Table 3. ABI channels and the corresponding Landsat channels utilized for Navigation (NAV) measurements. The ABI non-window IR channels are excluded from the NAV measurements (and not included in this table) because they cannot see the ground and so cannot be compared with Landsat chips

\begin{tabular}{|c|c|c|c|c|c|}
\hline ABI Channel & $\begin{array}{c}\text { ABI } \\
\text { Wavelength } \\
(\mu \mathrm{m})\end{array}$ & $\begin{array}{c}\text { ABI Nadir } \\
\text { Resolution } \\
\text { (km) }\end{array}$ & $\begin{array}{c}\text { Landsat } 8 \\
\text { Band }\end{array}$ & $\begin{array}{c}\text { Landsat } 8 \\
\text { Wavelength } \\
(\mu \mathrm{m})\end{array}$ & $\begin{array}{c}\text { Landsat } 8 \\
\text { Spatial } \\
\text { Resolution (m) }\end{array}$ \\
\hline 1 & $0.45-0.49$ & 1 & 2 & $0.45-0.51$ & 30 \\
\hline 2 & $0.59-0.69$ & 0.5 & 4 & $0.64-0.67$ & 30 \\
\hline 3 & $0.846-0.885$ & 1 & 5 & $0.85-0.88$ & 30 \\
\hline 5 & $1.58-1.64$ & 1 & 6 & $1.57-1.65$ & 30 \\
\hline 6 & $2.225-2.275$ & 2 & 7 & $2.11-2.29$ & 30 \\
\hline 7 & $3.80-4.00$ & 2 & \multirow{4}{*}{10} & \multirow{4}{*}{ 10.60-11.19 } & \multirow{4}{*}{100} \\
\hline 11 & $8.3-8.7$ & 2 & & & \\
\hline 13 & $10.1-10.6$ & 2 & & & \\
\hline 14 & $10.8-11.6$ & 2 & & & \\
\hline 15 & $11.8-12.8$ & 2 & \multirow{2}{*}{11} & \multirow{2}{*}{$11.50-12.51$} & \multirow{2}{*}{100} \\
\hline 16 & $13.0-13.6$ & 2 & & & \\
\hline
\end{tabular}
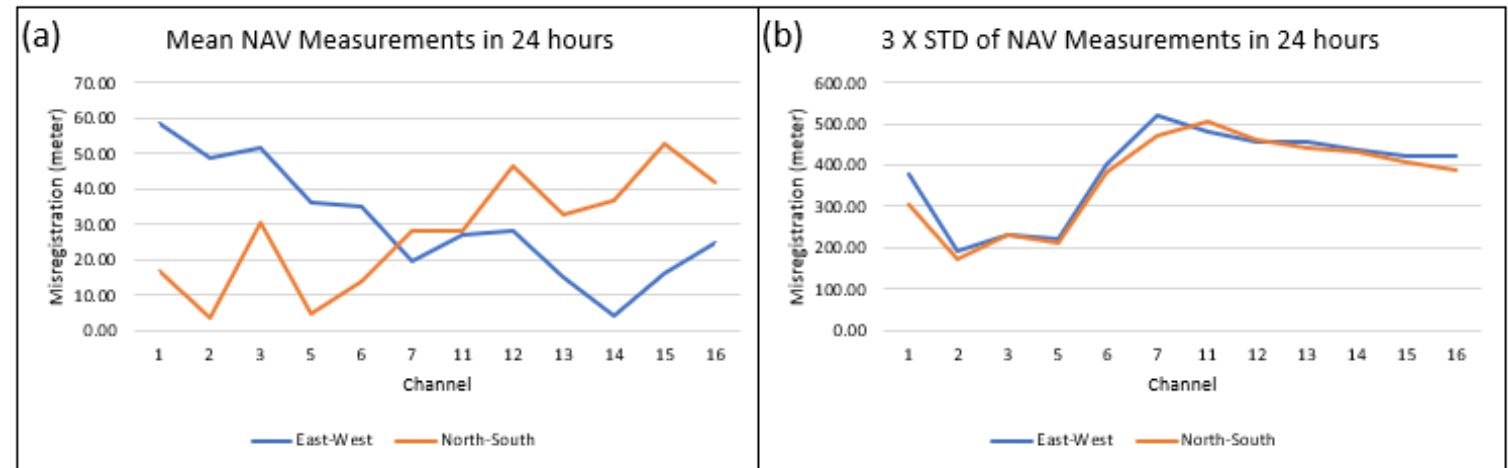

Figure 14. The mean (a) and 3 times standard deviation (b) of navigation error of a 24-hour period (June 1st 18:00 UTC to June 2nd 17:59 UTC 2019) for GOES-16. The misregistration is converted to nadir equivalent distance from the angular space of the fixed grid projection. 
The frame-to-frame registration error is assessed by direct matching between successive ABI images in the same spectral channel. There are more than 1000 evaluation locations over the ABI full disk where subset images are extracted from one image and used as templates for matching with the other. The average misregistration of the good-quality, clear-sky matches in a scene is considered to be the misregistration of the scene pair. The most recent ABI frame-to-frame registration accuracy is within 25 meters at nadir except non-window IR channels (Figure 15), which cannot be accurately assessed in this manner.
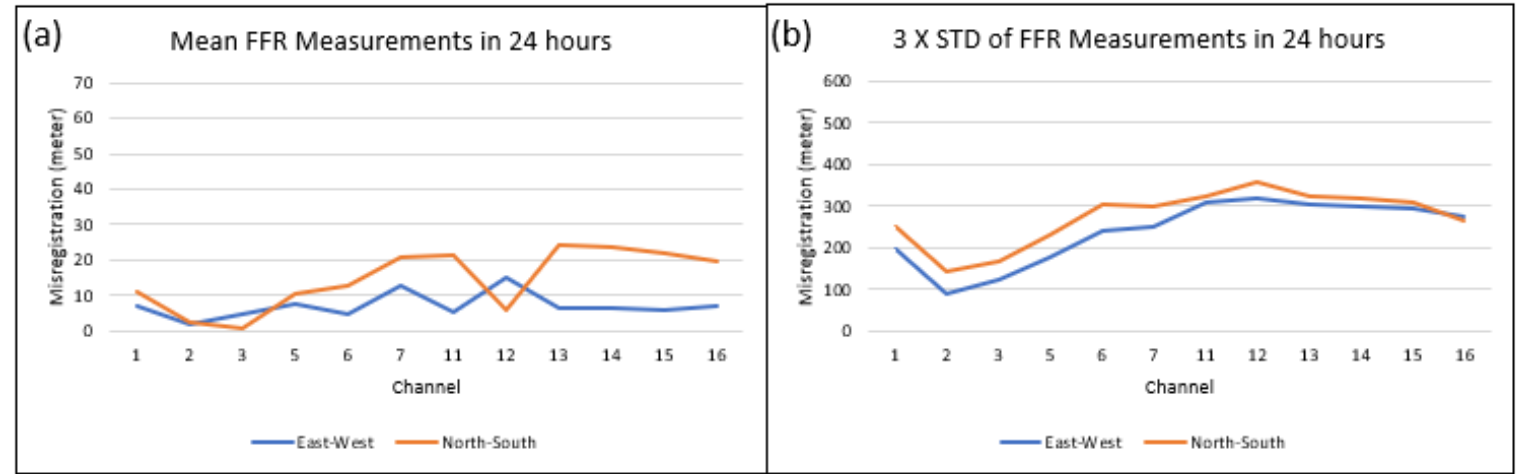

Figure 15. The mean (a) and three times standard deviation (b) of frame-to-frame error of a 24-hour period (June 1st 18:00 UTC to June 2nd 17:59 UTC 2019) for GOES-16. The misregistration is converted to nadir equivalent distance from the angular space of the fixed grid projection.

The results presented above are typical of GOES-16 INR performance. GOES-17 INR performance has similar characteristics. Both satellites satisfy their mission INR requirements for navigation $(28 \mu \mathrm{rad}$ or $1 \mathrm{~km}$ at the subsatellite point, 3-sigma) and frame-to-frame registration ( $21 \mu \mathrm{rad}$ or $0.75 \mathrm{~km}$ at the subsatellite point, three-sigma, for channels with $<2 \mathrm{~km}$ resolution and $28 \mu \mathrm{rad}$ or $1 \mathrm{~km}$ for the others) with substantial margins. INR performance is subject to continuous monitoring and improvement through the efforts of the GOES-R program.

\subsubsection{Remapped MODIS Granules}

The IPATS technology can also be used to validate the geometric accuracy of the MODIS granules after they have been remapped into the GOES fixed-grid projection, verifying both the quality of the navigation of the MODIS pixels and the correctness of the remapping process. Any residual systematic errors can be measured by matching with Landsat 8 chips, exactly as just described. Any systematic residual error that can be measured can also be compensated by making an adjustment to the remapping and redoing it. Figure 16 shows an example of a remapped Aqua granule with the outlines of the Landsat 8 chips used in the verification. The error statistics of good quality matches (peak correlation coefficient $>0.9$ ) in this case are means of -0.06 pixels (EW) and -0.03 pixels (NS), and standard deviations of 0.09 pixels in both directions. The same statistics for the ABI B02 CONUS scene most simultaneous with this granule are means of -0.14 pixels (EW) and -0.03 pixels (NS), and standard deviations of 0.06 pixels in both directions. 


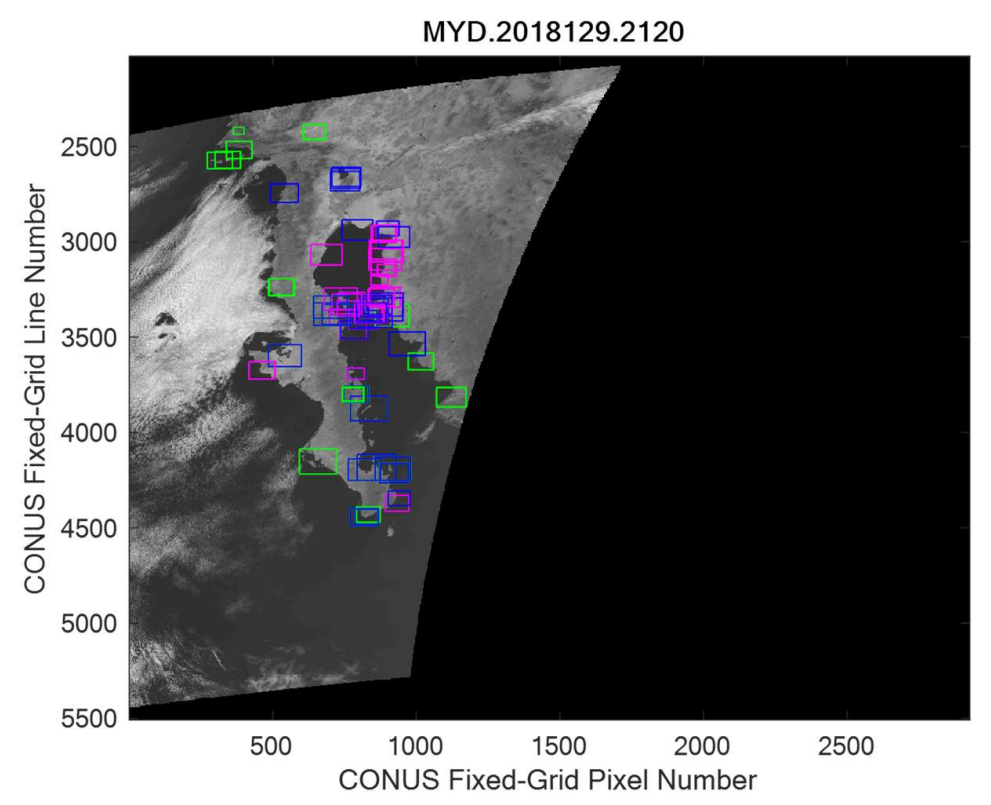

Figure 16. The performance assessment tool set (IPATS) Landsat 8 templates can measure any residual geometric error in the remapped MODIS granule. The outlines are color-coded in accordance with the normalized cross correlation (NCC) peak value (magenta $>0.95$, blue $>0.9$, and green $>0.5$ ).

\section{Results and Discussion}

\subsection{Validation}

As explained in Section 2.2.1, the algorithm to jointly retrieve AMVs and stereo heights is identical to that of our previous MISR-GOES study [9]. Here we first validate the extension of the algorithm to MODIS and GOES by comparing our MODIS-GOES results with MISR-GOES 3D-winds and NOAA operational wind products. The former is possible only for MODIS on Terra, since MISR does not fly on Aqua, and only for the reflective channels because MISR lacks thermal IR capabilities. Figure 17 shows MISR Path 24 as it crosses MODIS granule MOD2018196.1700.061 over the Great Plains of the United States. Where there is common coverage, we can make a direct comparison. Each MODIS-GOES retrieval site is paired with the nearest MISR-GOES retrieval site to make the comparison between the MODIS-GOES and MISR-GOES retrievals. Because the templates in the MODIS-GOES case are taken from the GOES ABI fixed-grid projection, and in the MISR-GOES case, templates are taken from the MISR swath mapped into a Space Oblique Mercator projection, there cannot be a perfect comparison with identical pattern content in the respective templates. On the other hand, the comparison is simultaneous, and it is useful for confirming the MODIS-GOES retrievals. The results of a comparison can be seen in Figure 18 for the winds and Figure 19 for the retrieved heights. 



Figure 17. A MODIS granule that has been remapped into the GOES ABI fixed-grid projection is overlaid with red boxes at the left (a) showing the blocks in the MISR path intersecting the granule. The MISR-GOES 3D-wind product is shown at the right (b). The MODIS granule is MOD2018196.1700.061 (MODIS Band 1) and is simultaneous with MISR Path 24, Orbit 98797. (Note: retrieval sites are thinned 4:1 in each direction for clarity).
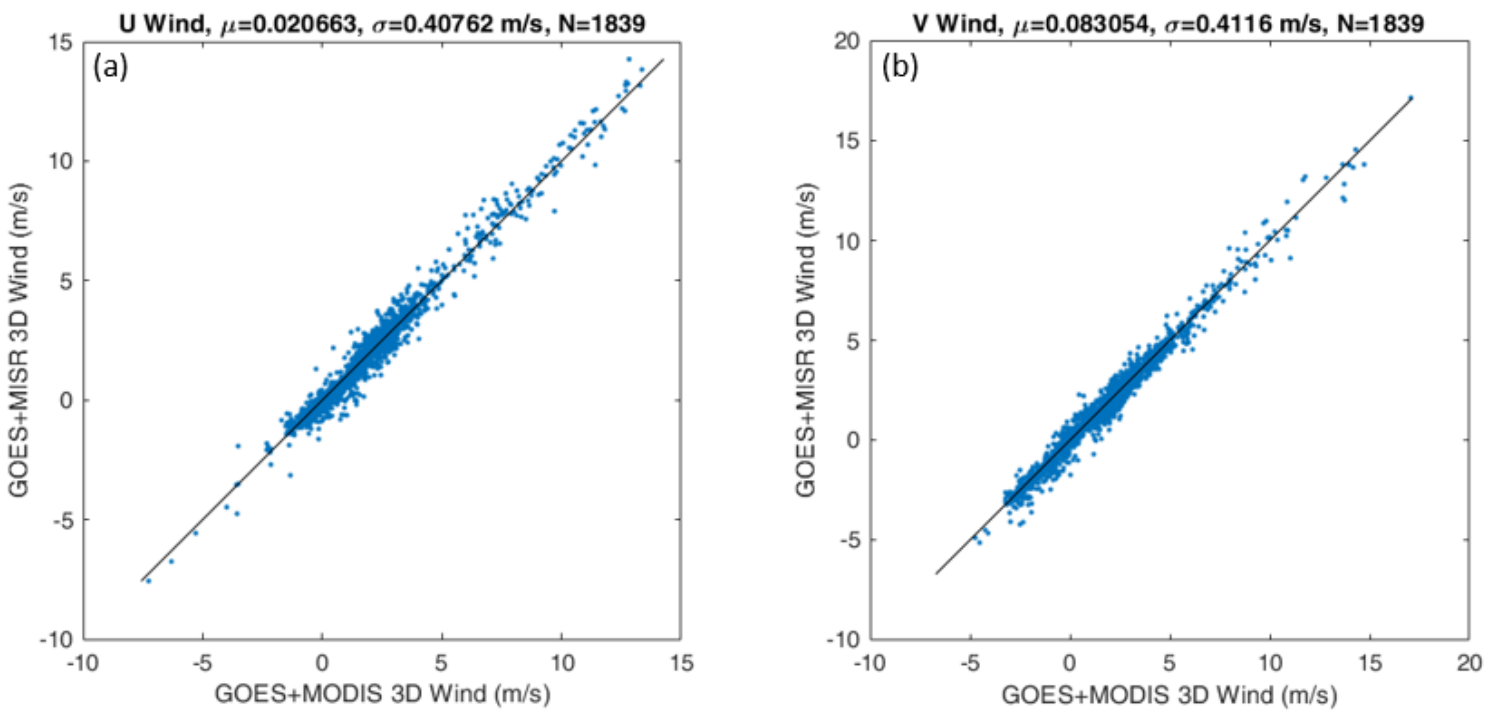

Figure 18. Retrieved winds from MODIS and GOES are compared to retrievals from MISR and GOES in the U (+East) and V (+North) directions on the tangent plane to the WGS84 ellipsoid in (a) and (b) respectively. The diagonal line is the perfect match, and robust statistics (mean $\mu$ and standard deviation $\sigma$, over sample size $\mathrm{N}$ ) are computed over the retrieval differences. The Median Absolute Difference (MAD) filter technique is used with a six-sigma threshold to edit out spurious pairings then the regular mean and standard deviation are computed over the remaining population. The MODIS granule is MOD2018196.1700.061 (MODIS Band 1) and is simultaneous with MISR Path 24, Orbit 98797. 


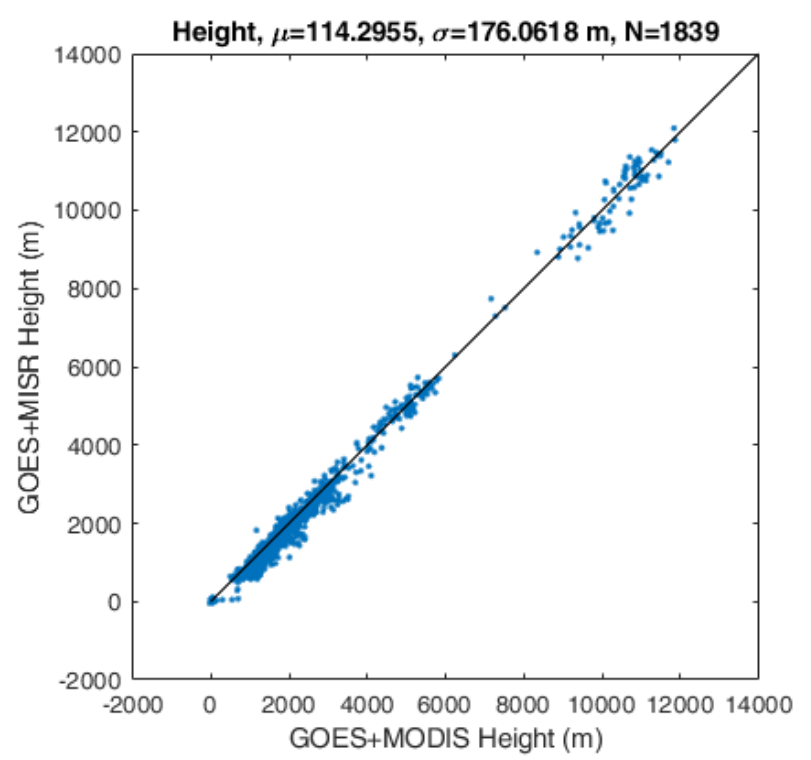

Figure 19. Jointly retrieved wind heights are compared between the MODIS-GOES and MISR-GOES cases with robust statistic on their differences (mean $\mu$ and standard deviation $\sigma$, over sample size $\mathrm{N}$ ). The heights are with respect to the WGS84 ellipsoid. The MODIS granule is MOD2018196.1700.061 and is simultaneous with MISR Path 24, Orbit 98797.

The comparison between the wind velocities shows small biases and standard deviations that are in accord with an estimated wind accuracy of $<0.5 \mathrm{~m} / \mathrm{s}$ for the MISR-GOES product [9]. This is supported by a comparison between the MODIS-GOES winds and the NOAA operational derived motion wind (DMW) product for the same time as shown on Figure 20. This should not be surprising since the same GOES-16 CONUS scenes are used for both. There are fewer samples in the DMW comparison because the NOAA operational winds are generally less densely sampled. Paired sites that are too far away $(>2.2 \mathrm{~km})$ are not considered coincident and therefore not part of the comparison.
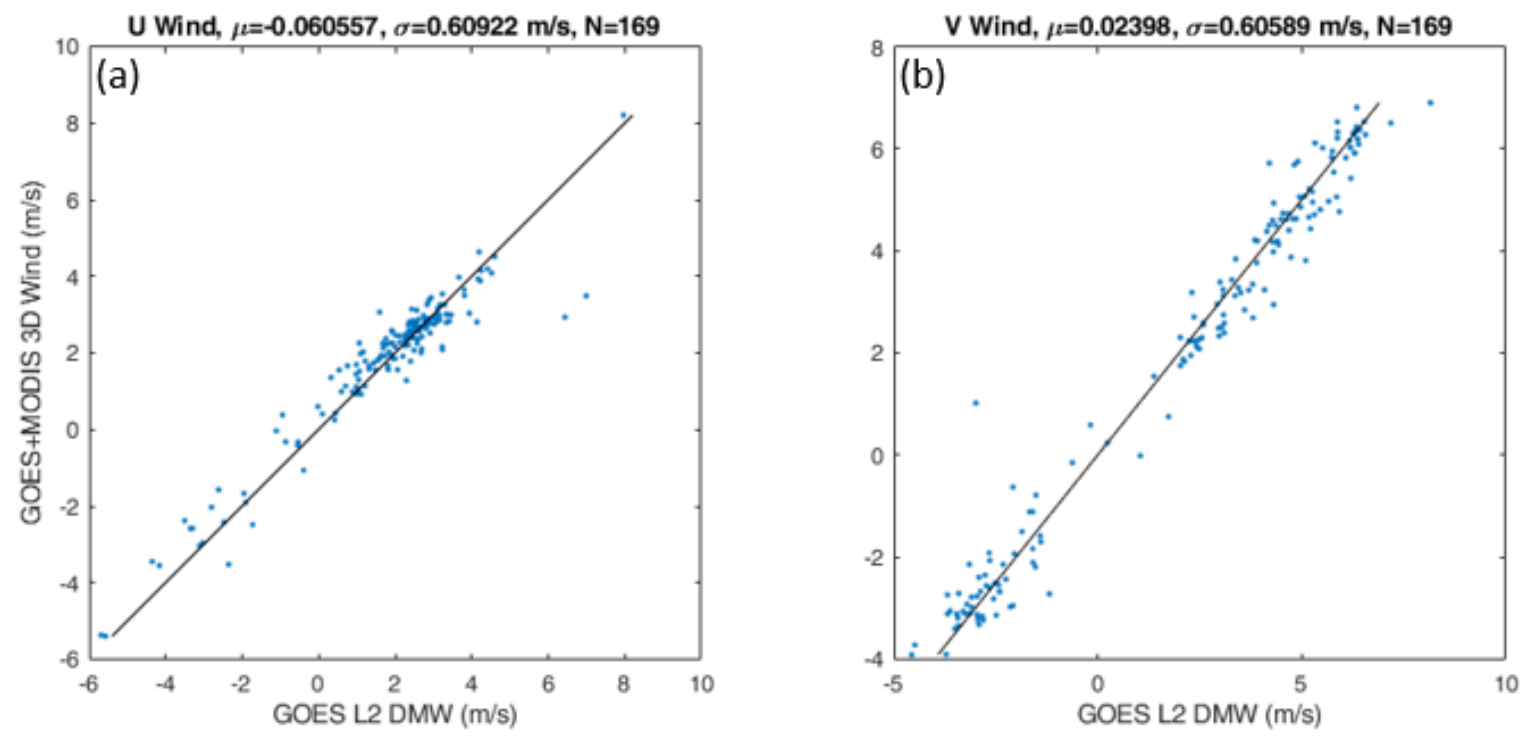

Figure 20. Retrieved MODIS-GOES winds are compared with the NOAA operational derived motion wind (DMW) product along the $U$ axis (a) and $V$ axis $(\mathbf{b})$. The diagonal line is the perfect match, and the statistics are computed over the retrieval differences (mean $\mu$ and standard deviation $\sigma$, over sample size N). The MODIS granule is MOD2018196.1700.061 and the DMW product is for ABI band 2 and the CONUS scene with product time 2018/196 17:02. 
A similar comparison between MODIS-GOES 3D-wind retrievals and the operational DMWs is shown in Figure 21 for ABI Band 14. The IR spatial resolution is four times coarser than that of ABI Band 2.
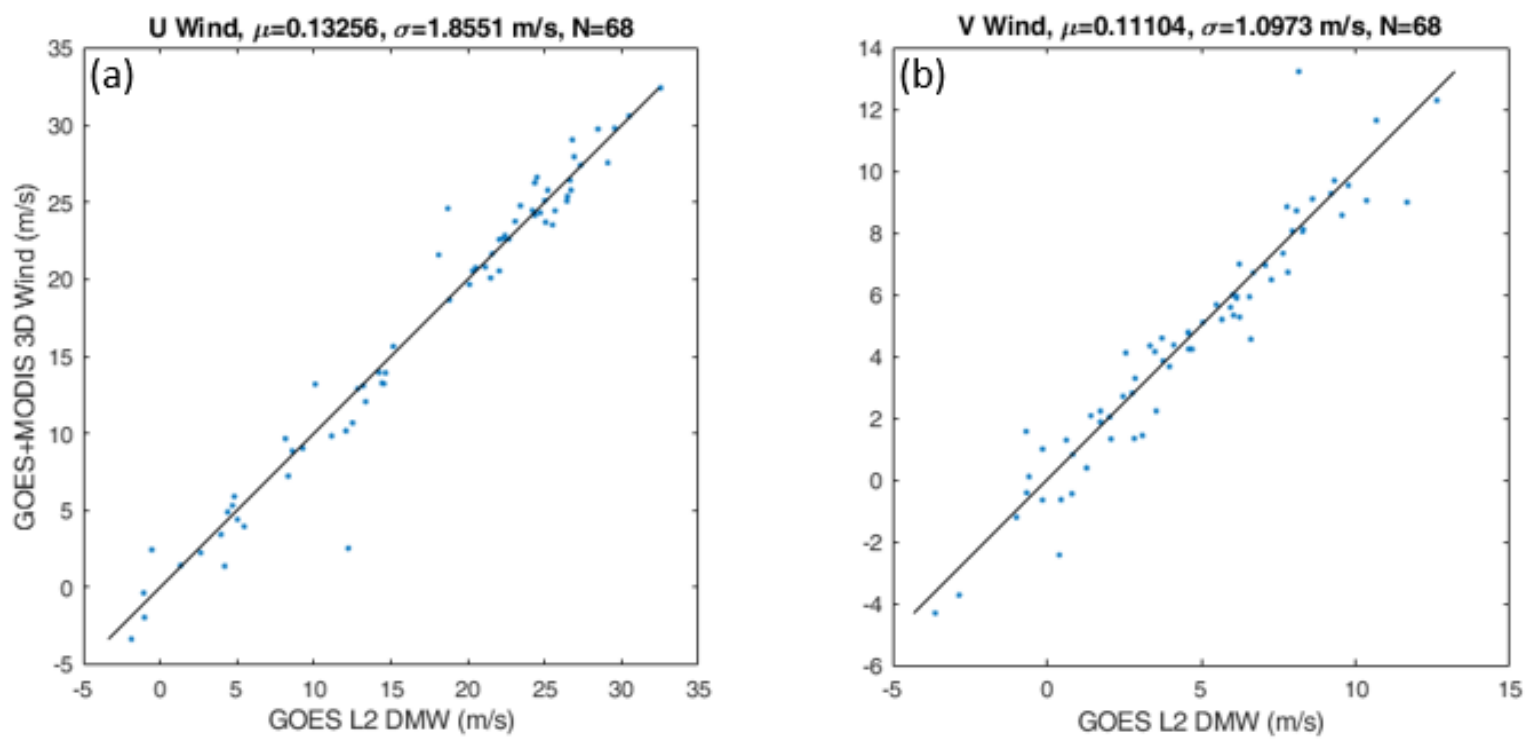

Figure 21. Retrieved MODIS-GOES winds are compared with the NOAA operational DMW product for an IR case along the $U$ axis (a) and $V$ axis (b). The diagonal line is the perfect match, and the statistics are computed over the retrieval differences (mean $\mu$ and standard deviation $\sigma$, over sample size N). The MODIS granule is MOD2019057.2000.061 and the DMW product is for ABI band 14 and the CONUS scene with product time 2019/057 20:02.

The operational NOAA DMW wind vectors are assigned pressure levels. As noted in [9], when converted to an equivalent height in atmosphere, the differences between the MISR-GOES 3D-wind height assignments and the DMW height assignments can be as large as $1 \mathrm{~km}$. A more accurate validation of the MODIS-GOES height assignments is made by looking at the clear-sky retrievals from the MODIS-GOES 3D-winds. This is a method that has been pioneered with MISR [21]. It is true that the ground retrievals are not cloud tracks and therefore not winds; however, the range of terrain heights above the ellipsoid can be several $\mathrm{km}$, a good range for low clouds, and the fact that the ground is known to be static also offers a means to obtain an estimate of the velocity uncertainty for zero-speed objects. Figure 22 is an example of ground-point retrievals from MODIS-GOES 3D-winds. The clear-sky ground retrievals are classified by whether the retrieval site is over land, apparently close to the ground $(<300 \mathrm{~m}$ ), and stationary (within $0.3 \mathrm{~m} / \mathrm{s}$ of zero in both $\mathrm{U}$ and $\mathrm{V}$ axes) and the height error is the difference between the retrieved height and the known terrain height. For the purposes of calculating the velocity errors, the velocity limit is relaxed to $2 \mathrm{~m} / \mathrm{s}$ to admit possibly larger errors. In the case shown, there is a small height bias of $18 \mathrm{~m}$. The equivalent ground-point height bias for the MISR-GOES ground-point retrievals (case shown in Figure 17) is $-47 \mathrm{~m}$ with a $162 \mathrm{~m}$ standard deviation. Therefore, the relative height bias is $65 \mathrm{~m}$, which is smaller but still comparable with the mean difference seen in Figure 19. The small bases may be due to small residual geolocation errors in the three systems. The error statistics in Figure 22 are also comparable with the uncertainties from the state covariance matrix that are shown in Figure 10 for the same case. The height retrieval errors will depend on the LEO-GEO relative viewing geometry and will become large near the singular geometry as described in Section 2.1.3. The uncertainty from the covariance is an effective guide to retrieval quality. 

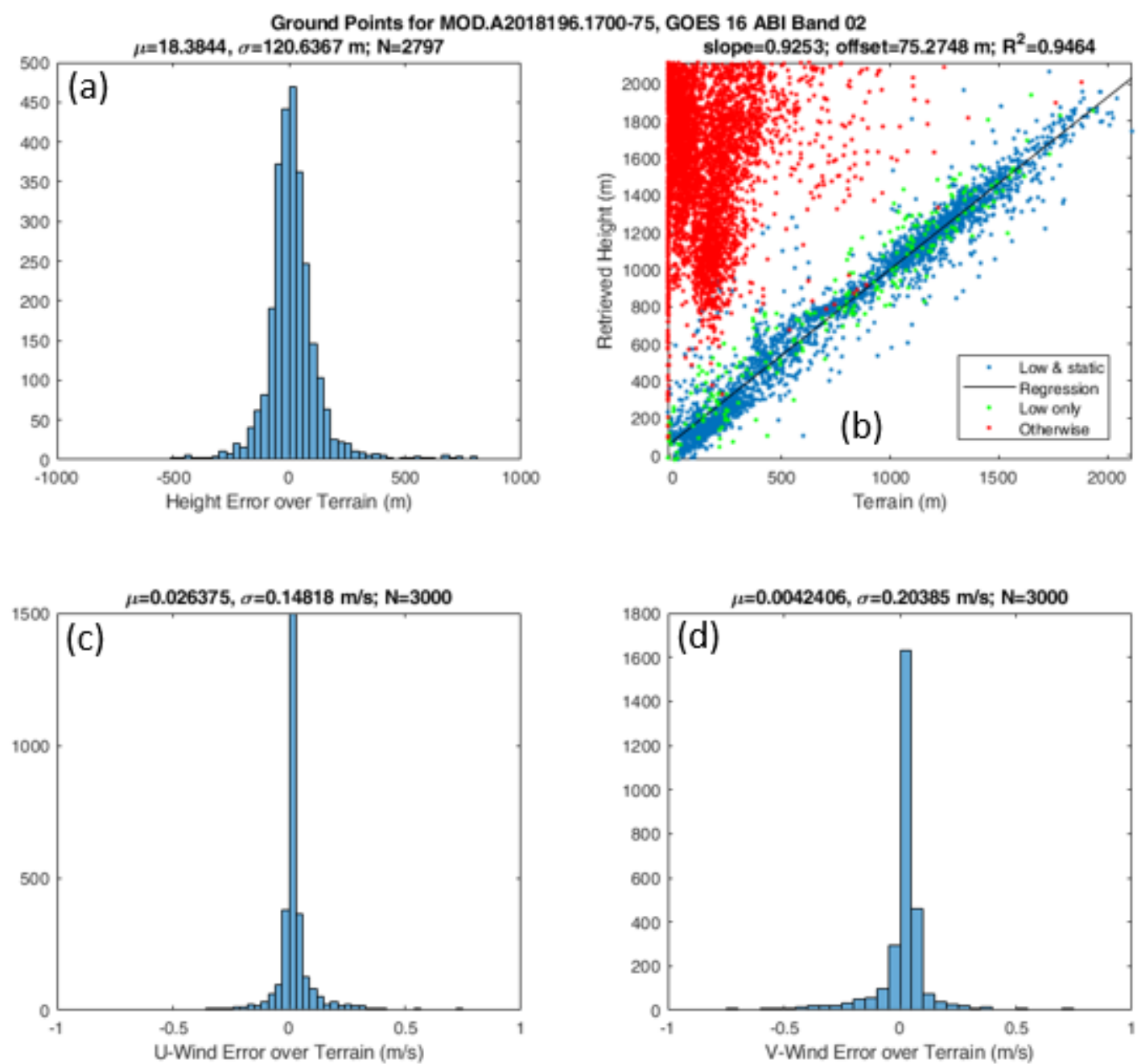

Figure 22. Clear-sky ground retrievals from the MODIS-GOES 3D-winds show the accuracies of retrieved heights (a) and velocities $(\mathbf{c}, \mathbf{d})$. The mean $\mu$ and standard deviation $\sigma$ over sample size $\mathrm{N}$ are computed for each histogram and the regression parameters (b) for retrieved height versus terrain height are reported for the ground-point class ("Low and static"). The MODIS granule is MOD2018196.1700.061 (MODIS band 1).

Thermal-IR ground point measurements can sometimes be obtained for a granule (Figure 23). In the thermal IR, the pixel size is four times $(2 \mathrm{~km}$ versus $0.5 \mathrm{~km})$ that of the previous example, which results in larger errors in the height assignment and static-target velocity error. 
Ground Points for MYD.A2019057.2000-75, GOES 16 ABI Band 14

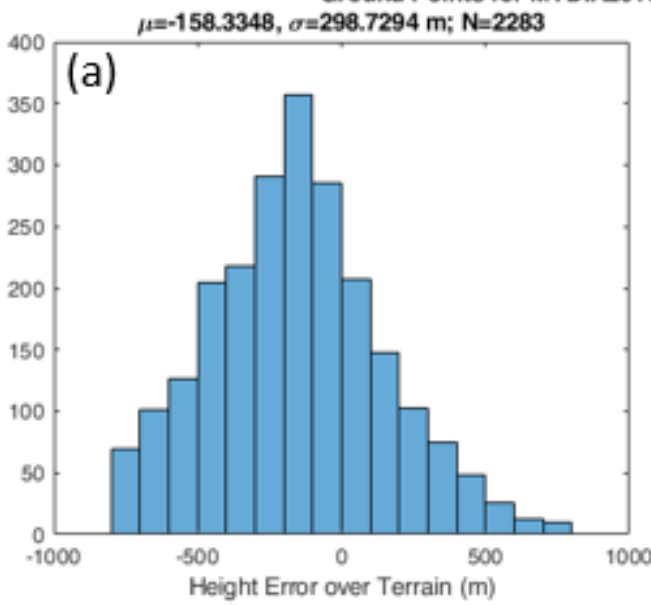

slope $=0.934 ;$ offset $=-81.0236 \mathrm{~m} ; R^{2}=0.83077$
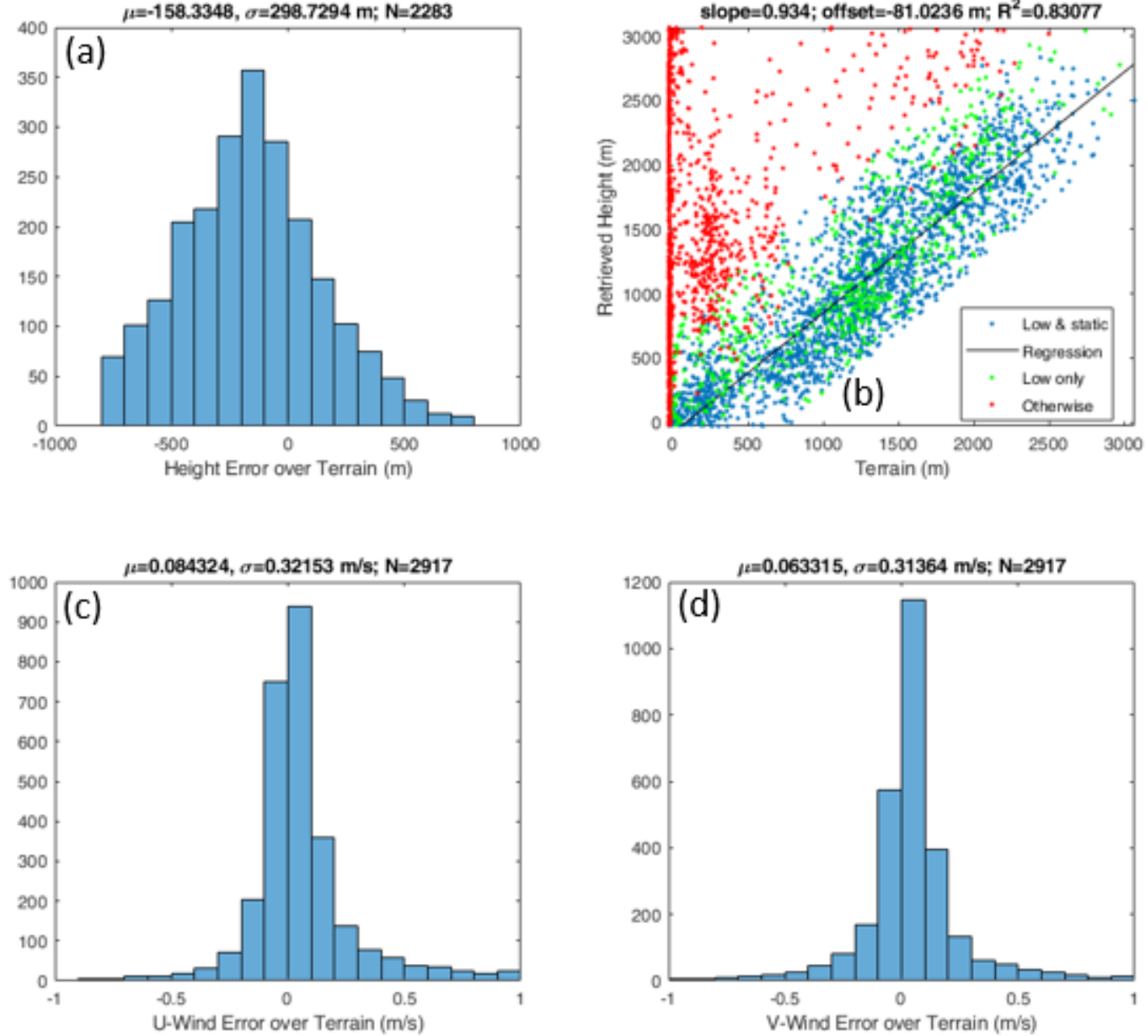

Figure 23. Clear-sky ground retrievals from the MODIS-GOES 3D-winds show the accuracies of retrieved heights (a) and velocities (c,d) for an infrared (IR) case. The mean $\mu$ and standard deviation $\sigma$ over sample size $\mathrm{N}$ are computed for each histogram and the regression parameters (b) for retrieved height versus terrain height are reported for the ground-point class ("Low and static"). The MODIS granule is MOD2019057.2000.061 paired with ABI Band 14, CONUS scene time 2019/057 20:02.

In summary, we compare MODIS-GOES retrievals to simultaneous collocated MISR-GOES retrievals using the same 3D-winds retrieval algorithm. In our previous work [9], we compared MISR-GOES retrievals to NOAA operational DMWs and extensively analyzed ground-point retrievals to validate the application of the new algorithm with MISR and GOES datasets. We concluded that MISR-GOES retrieval height errors were $<200 \mathrm{~m}$ and AMV errors $<0.5 \mathrm{~m} / \mathrm{s}$. The MODIS-GOES to MISR-GOES comparison above (Figures 18 and 19) shows an agreement to within these error bounds. Our previous work also compared the MISR-GOES retrievals to NOAA operational DMWs that use IR methods for height assignments. The two often differed by $\sim 1 \mathrm{~km}$. Therefore, we expect that both the MISR-GOES and MODIS-GOES retrievals (except near the singular geometry) should improve the state of the art in AMV height assignments if not necessarily the AMVs alone. However, since wind speed can vary significantly with altitude, a better height assignment can also mean better correlation between the AMV and the in situ wind. Ground-point retrievals are another powerful method for validation. The results presented above (Figure 22) are consistent with those from the MODIS-GOES to MISR-GOES comparison. The processing of a ground pattern is identical to that of a cloud pattern and a range of heights can be sampled according to the natural variation of the terrain. The fact that ground points are static could be a limitation, but comparisons with other AMV products seems perfectly 
adequate to validate retrieved AMVs alone. The true height assignment for an AMV is, of course, more nuanced as a tracked pattern is not necessarily confined to a single plane. Using the same pattern used for AMV determination to measure directly the pattern height through parallax is a more direct method of assigning a height than IR methods, but certainly additional validations using in situ wind measurements would be beneficial to quantify the relative benefits of the new methods.

\subsection{Applications}

The MODIS-GOES algorithm greatly enlarges the spatiotemporal coverage of the stereo technique using LEO-GEO pairs, compared with the MISR-GOES algorithm developed in our earlier study [9]. Vector winds are essentially retrieved from a triplet/doublet of rapidly refreshed GEO images with the stereo height being observable thanks to the snapshot provided by the LEO satellite at a different view angle and interposed in the GEO image sequence. The newly extended algorithm requires no synchronization between the LEO-GEO pair, only the ability to time tag pixels, because the parallax solver can account for feature motion effects if the LEO image is not taken in synchronization with the GEO images.

The wider spatial coverage from MODIS-like imagers and frequency of LEO-GEO pairings allow a host of science applications that require accurate knowledge of AMVs and their heights, including PBL processes, overshooting convective clouds, and dispersion of wildfire smoke plumes. Following the successful development of the MODIS-GOES 3D-wind algorithm, we demonstrate here its capabilities with several applications, to illustrate the future research that the stereo-AMV technique will enable.

\subsubsection{Quasi-3D Representation of Mesoscale Dynamics (January 2019)}

The MODIS-GOES algorithm can retrieve an AMV field with $\sim 4 \mathrm{~km}$ horizontal resolution and $<300 \mathrm{~m}$ vertical accuracy. Since AMVs are derived from cloud-top features, each AMV represents a vector at a height characteristic of the cloud tops. In a scene of broken clouds with tops at different altitudes, a densely sampled, high-resolution AMV field can effectively sample multiple layers. Figure 24a illustrates a benefit of high-resolution $(<4 \mathrm{~km})$ AMV retrievals for representing mesoscale dynamics. Without thinning the AMV field from different vertical levels (as often practiced by NWP data assimilation), these AMVs can provide a quasi-3D view of mesoscale dynamics over a synoptic domain.

Figure $24 \mathrm{~b}$ is a case on January 31, 2019, when cold Arctic air descended into CONUS, creating a severe weather event with heavy snow and record cold in many Midwest states. The coldest temperature recorded by a ground station was $-49^{\circ} \mathrm{C}$ in Cotton, Minnesota, while it reached $-35^{\circ} \mathrm{C}$ in Rockford, Illinois, breaking its $-32{ }^{\circ} \mathrm{C}$ record set in January 1982. The AMV pattern retrieved from the MODIS-GOES algorithm over CONUS represents the classic cold air outbreak (CAO) case where a strong upper-level trough descends into mid-latitudes while low-level warm air collides with the trough, generating complex 3D dynamics. 




Figure 24. (a) Illustration of the improvement from high-resolution $(<4 \mathrm{~km})$ AMVs in representing a quasi-3D synoptic flow. Compared to conventional AMV retrievals at a coarser resolution, the high-resolution algorithm provides more vertical levels of AMVs that can be assimilated in the NWP system. (b) AMVs at $2 \mathrm{~km}$ horizontal resolution from the MODIS-GOES retrieval on January 31, 2019 when a cold Arctic system dipped down to CONUS and created severe winter storms in most of the northern states (c). The height of wind vectors is color coded and shows a range of AMV heights from the surface to $\sim 11 \mathrm{~km}$. The blue lines indicate the borders of MODIS image granule in this case (as shown in the embedded image).

\subsubsection{PBL Dynamics (January-February 2019)}

The primary motivation for developing the MODIS-GOES 3D-wind algorithm is to provide a better height assignment for AMVs. PBL AMVs are one of the challenging cases for height assignments, primarily because traditional IR methods have difficulty with the PBL thermodynamic structure, which may include inversions. The stereo approach as implemented in the MODIS-GOES algorithm does not require any knowledge about atmospheric thermal structures. Therefore, AMV retrievals in the PBL provide a good test for the new algorithm.

In Figure 24, the PBL AMVs and their structure are hardly seen due to the color scale used to include the high-level winds. To highlight the PBL winds, Figure 25 shows the same case with a different color scale for the region over Lakes Superior and Michigan where the CAO event produced a very dynamic PBL cloud field. As the cold air blows over open water, convective activity in the marine PBL produces cloud streets that can often stretch hundreds of kilometers [22]. These organized cloud patterns occur more than $50 \%$ of the time over the North Atlantic during winter, and most of them last less than 2 days [23]. These clouds can also occur over an extensive lake surface, such as in the Great Lakes (Figure 25).

On January 31, 2019, most of Lake Superior and Lake Michigan still had open water except near the shore where lake ice might have formed. The rising PBL top is readily seen in the AMV height retrievals, with the colored wind vectors in Figure 25 showing that the PBL height increases steadily from $\sim 400 \mathrm{~m}$ on the western shore to $\sim 1100 \mathrm{~m}$ on the eastern shore of Lake Michigan. The CAO event over Lake Michigan was dominated by the zonal wind, which was approximately constant at $\sim 8 \mathrm{~m} / \mathrm{s}$ for these PBL heights. The rise in PBL height is caused by growth of a stably stratified boundary layer, downstream from the land/ice edge, which progresses slowly by advection of continental air over the water (Figure 26). As detailed by Garratt [24], the depth of the PBL layer is a function of distance and wind speed. PBL clouds from CAO events have an important implication for Earth's weather and climate systems, because they can cause heavy snowfall and modulate shortwave (SW) solar radiation.

The 3D-wind algorithm provides an additional method for cloud detection during day and night. Radiometric methods for cloud detection would encounter a great difficulty for the low clouds over a white snowy/icy background such as in Figure 25. With the stereo AMV product, we can provide more confidence for cloud detection by classifying the surface retrievals as low-wind-speed $(<0.5 \mathrm{~m} / \mathrm{s})$ and 
low height $(<300 \mathrm{~m})$ features. This has a clear benefit for those applications (e.g., snow and ice cover) that require a robust cloud mask.

The wind direction retrieved from the 3D-wind algorithm represents an instantaneous direction for a wind vector. Although the cloud streets from the CAO event are generally in line with the wind vectors, their patterns reflect a history of wind transport. Evolution of PBL height can cause the clouds streets not to align with the instantaneous wind direction. This has an implication for the semi-autonomous tool (MISR INteractive eXplorer, or MINX) used for MISR-only PBL AMV retrievals. The MINX retrievals require a priori information about the wind direction in order to mitigate the aliasing problem between along-track motion and height parallax. The MINX operators usually use the cloud streets as a guess for the wind direction. As revealed in the MODIS-GOES 3D-wind AMVs in Figure 25, the cloud streets are not necessarily same as the instantaneous wind direction in this scene.

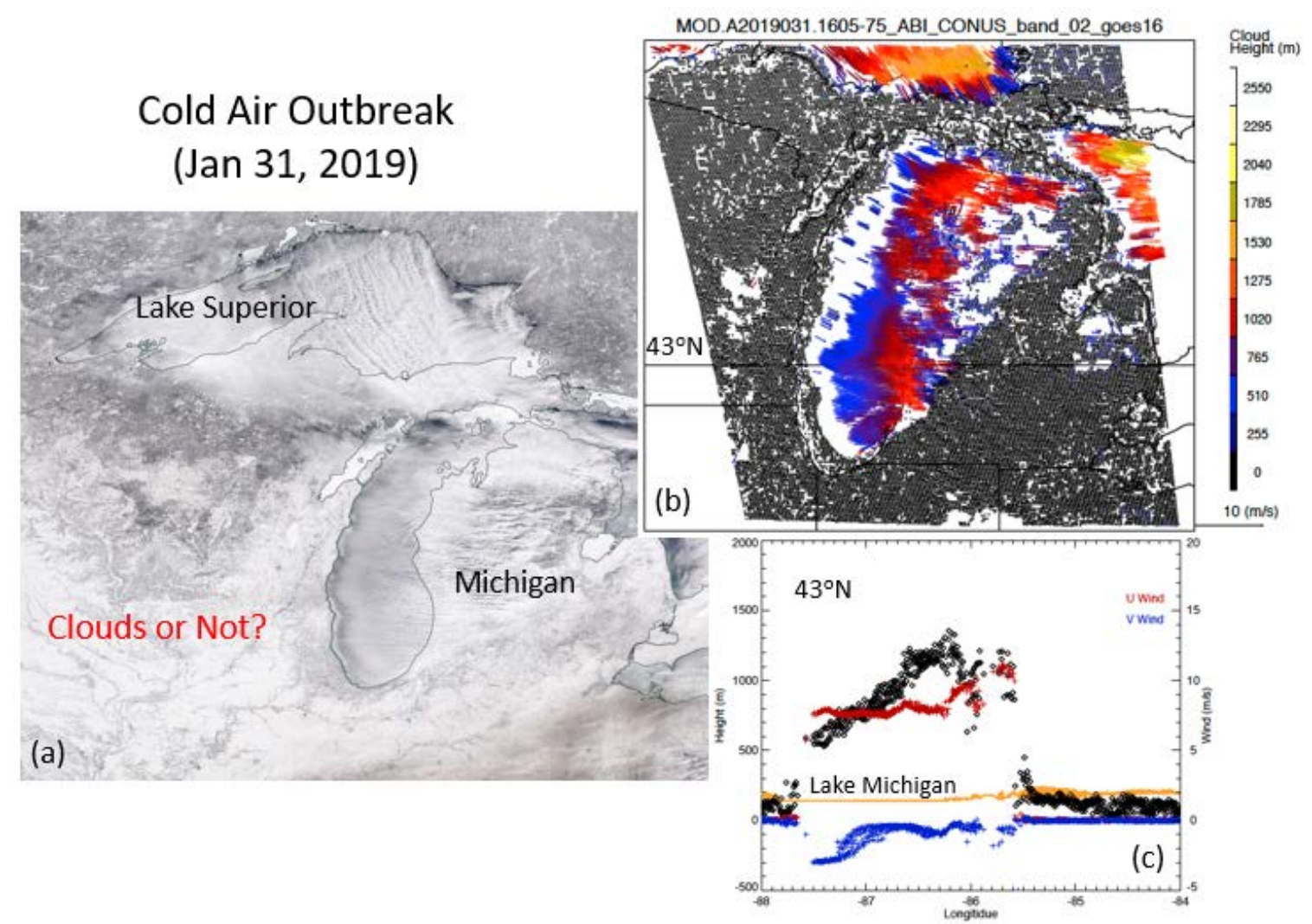

Figure 25. PBL cloud streets are readily seen from the cold air outbreak (CAO) event (a) over Lakes Superior and Michigan (shown at larger scale in Figure 24). The bottom-right panel (c) is a cross-section of the full set of retrievals (b) at $43^{\circ} \mathrm{N}$ latitude where terrain height is in orange and the stereo height is in black, showing PBL clouds rising in height from $400 \mathrm{~m}$ to $1100 \mathrm{~m}$ over Lake Michigan, as cold air blows over the relatively warmer water. Heavy snow often occurs downstream from the lake, as a result of the so-called 'lake effect'. 


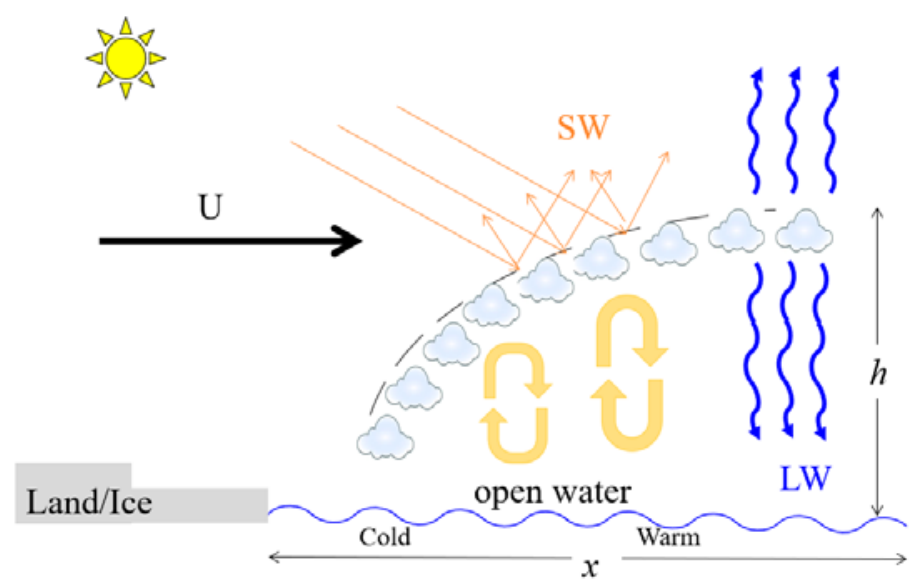

Figure 26. A schematic diagram to illustrate interactions between cold air and warmer water in producing a rising boundary-layer top over open water during the CAO event.

The direction of PBL AMVs can change in a short period of time. On February 1, 2019, the PBL AMVs veered northward in a narrow band over Lake Michigan (Figure 27). There was a less variation in the AMV height, as compared to the January 31, 2019 situation. Clouds and snowy/icy surfaces cannot be readily classified in the MODIS visible image but can with the stereo height map. It is evident that lake ice had grown along the shore during January 28-February 1, as the cold air froze more lake surface. The open water area plays an important role in forming the PBL clouds. In a recent study [25], we found using MISR that the PBL cloud amount increased significantly over the Arctic Ocean as a result of there being more open water from the summertime warming.
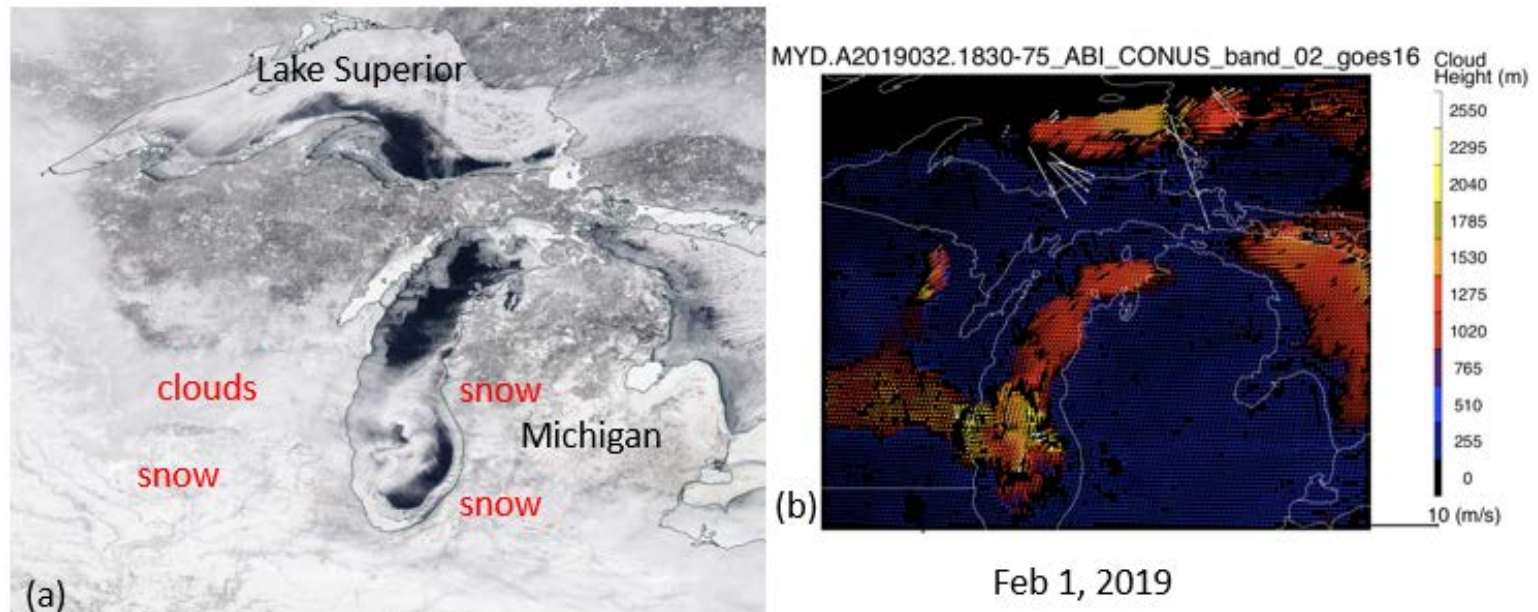

Feb 1, 2019

Figure 27. As in Figure 25 but for Feb.1, 2019 when the winds over Lake Michigan (a) changed their direction drastically and the PBL clouds were aloft at the approximately same altitude (b).

\subsubsection{Hurricane Michael (October 2018)}

The MODIS-GOES 3D-wind algorithm is applied to Hurricane Michael on October 9, 2018 to evaluate its AMV and stereo height retrievals from different spectral bands. As seen in Figure 28, shortly before the hurricane reached its peak intensity, MODIS on Terra flew over the eye at 16:25Z with full coverage across the span of the cyclone in the Gulf of Mexico. Hurricane Michael was a deadly Category 5 hurricane, known for its rapid intensification from category 2 on October 8 to category 5 on October 9 before its landfall on October 10. 


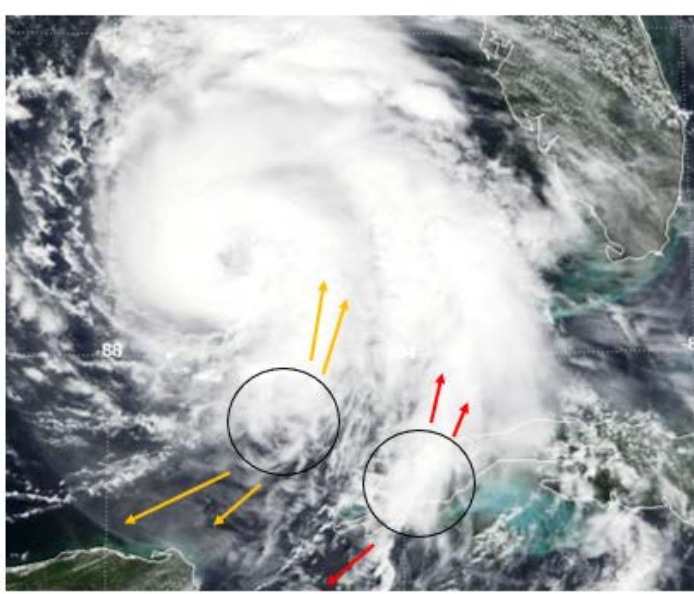

(a)

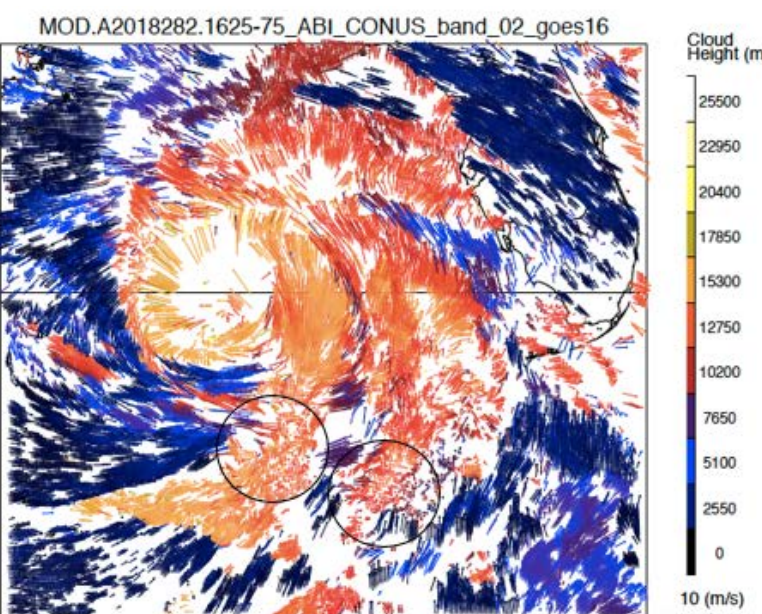

(b)

Figure 28. (a) MODIS image of Hurricane Michael on October 9, 2018 with the circles indicating deep convective zones and the arrows for wind direction. (b) Retrieved AMVs from ABI band 2 with height in color. The circles highlight small AMV wind speeds at high altitudes.

Retrieval experiments were conducted with different configuration parameters to evaluate the trade-off between AMV quality and spatial resolution in the Hurricane Michael case where Terra/MODIS are matched to GOES-16 CONUS images. A higher spatial resolution is always desired for mesoscale dynamics such as hurricanes and other severe weather phenomena, which requires a smaller chip size (i.e., size of an image template for pattern matching). On the other hand, since clouds are non-rigid features and can change (grow or decay) in time, a smaller chip size may lead to a noisier product, due to less robust pattern recognition. In this study we tested three chip sizes $(8 \times 8,16 \times 16$ and $32 \times 32 \mathrm{~km}$ ) for four spectral bands, namely, ABI bands 2 (visible), 4 (near IR), 7 (mid-wave IR), and 14 (thermal IR) as shown in Table 4. For each test, the sampling interval is a half of the chip size, so more retrievals are being made at the higher resolutions as well and consuming more computer resources. Figure 28 shows the high-resolution ( $4 \times 4 \mathrm{~km}$ sampled) AMV retrievals from band 2 with a chip size of $8 \times 8 \mathrm{~km}$. In the band 2 pairing, GOES-16 has a pixel resolution (500 m), $4 \times$ better than bands 4,7 and 14, to match MODIS 250-m pixel resolution, which allows pattern matching to produce good results at the highest resolution among all MODIS-GOES spectral band pairs. To evaluate AMV retrieval quality, we compare each wind vector $(\vec{U})$ with the background wind $\left(\vec{U}_{b}\right)$ in a $40 \times 40 \mathrm{~km}$ domain centered at this AMV. The norm $\left\|\vec{U}-\vec{U}_{b}\right\|<0.2 \mathrm{~m} / \mathrm{s}$ is used as a "consistency" flag, and Table 4 summarizes the percentage of consistent AMV retrievals from each band for different chip sizes. For the hurricane case, $\mathrm{ABI}$ band 2 can still produce useful AMVs at $8 \mathrm{~km}$ resolution, whereas bands 4, 7 and 14 AMVs can only go down to $16 \mathrm{~km}$ resolution mainly because their pixel resolution is $4 x$ coarser than Band 2 .

Table 4. AMV Quality (i.e., consistency) for the hurricane Michael retrievals.

\begin{tabular}{ccccc}
\hline Chip Size (km) & Band 2 & Band 4 & Band 7 & Band 14 \\
\hline $8 \times 8$ & $93 \%$ & $22 \%$ & $21 \%$ & $22 \%$ \\
$16 \times 16$ & $97 \%$ & $95 \%$ & $92 \%$ & $95 \%$ \\
$32 \times 32$ & $99 \%$ & $99 \%$ & $99 \%$ & $99 \%$ \\
\hline
\end{tabular}

The high-resolution AMV and height retrievals provide extraordinary insights into severe weather systems such as Hurricane Michael. Divergence of the wind fields at the top of cyclone deck, as highlighted by the circles in Figure 28, is indicative of strong deep convection zones in the arms that were strengthening the cyclone. The highlighted high-level AMVs are associated with a low wind speed as expected for deep convection reaching a neutral buoyancy level in the upper tropopause. 
Outside the deep convective zone, the upper-level AMVs must flow away from the updraft region, as a result of the mass conservation law. Such deep convection may occur in other parts of the cyclone's arms, but is obscured by the cirrus outflows aloft.

In addition to the chip size sensitivity, spectral bands can have different sensitivities to AMVs, because cloud features seen by each band can vary significantly. Band 2 (visible) is less sensitive to thin cirrus than band 14 (thermal IR). In the case of multi-layer clouds covered by thin cirrus, band 2 tends to see through the cirrus and finds the feature pattern below, whereas band 14 tends to find the cirrus for pattern matching. This is clearly evident in Figure 29 where there are more low-level clouds in the Band 2 AMVs than in band 14. This type of multi-layer clouds is mostly seen on the eastern side of the cyclone, and an advantage with both band 2 and 14 AMVs is to provide two distinct vertical levels of mesoscale dynamics at the same location. On the other hand, band $4(1.37 \mu \mathrm{m})$ is known as the "cirrus" band, which uses water vapor absorption to screen out low-level clouds and surface features. As seen in Figure 29, the majority of its retrievals are high-level AMVs, some of which are higher than those from bands 2 and 14 near the hurricane center, but not many low-level AMVs are found except where the atmosphere is dry and clear. Band 7 (mid-wave IR) is a channel that has about same amount of radiance from the reflected sunlight as from the terrestrial emissions. It has been used for fire/hot-spot, daytime snow/ice detection, and low-level AMVs. In this hurricane case, its yield for AMVs is not as great as bands 2 and 14, but it has a balanced sampling in the multi-layer cloud scenes.


Figure 29. Distributions of the AMV height retrieval (chip size $32 \times 32 \mathrm{~km}$ ) for the four spectral bands (ABI bands 2, 4, 7, and 14 in panels (a-d) respectively). The vertical line is $87.5^{\circ} \mathrm{W}$ longitude.

Different AMV sensitivities by the spectral bands is further illustrated by the vertical distribution of height measurements. Along the meridian of $87.5^{\circ} \mathrm{W}$ longitude (Figure 30a), bands 4 and 14 produce a peak stereo height as high as 17.8 and $17.4 \mathrm{~km}$, respectively, compared with $16.3 \mathrm{~km}$ from Band 2. Again, the higher peaks are consistent with the better cirrus sensitivity from bands 4 and 
14. Since these high-level cirrus clouds are clearly above the tropopause, it is impossible for the conventional IR techniques to provide accurate height assignment. Also noticeable in Figure 30a is the smoothness of band 2 and band 14 height measurements. Because these bands are spectral windows, cloud features are less attenuated by atmospheric emissions (e.g., $\mathrm{CO}_{2}$ and $\mathrm{H}_{2} \mathrm{O}$ ) and have better contrast for pattern recognition.
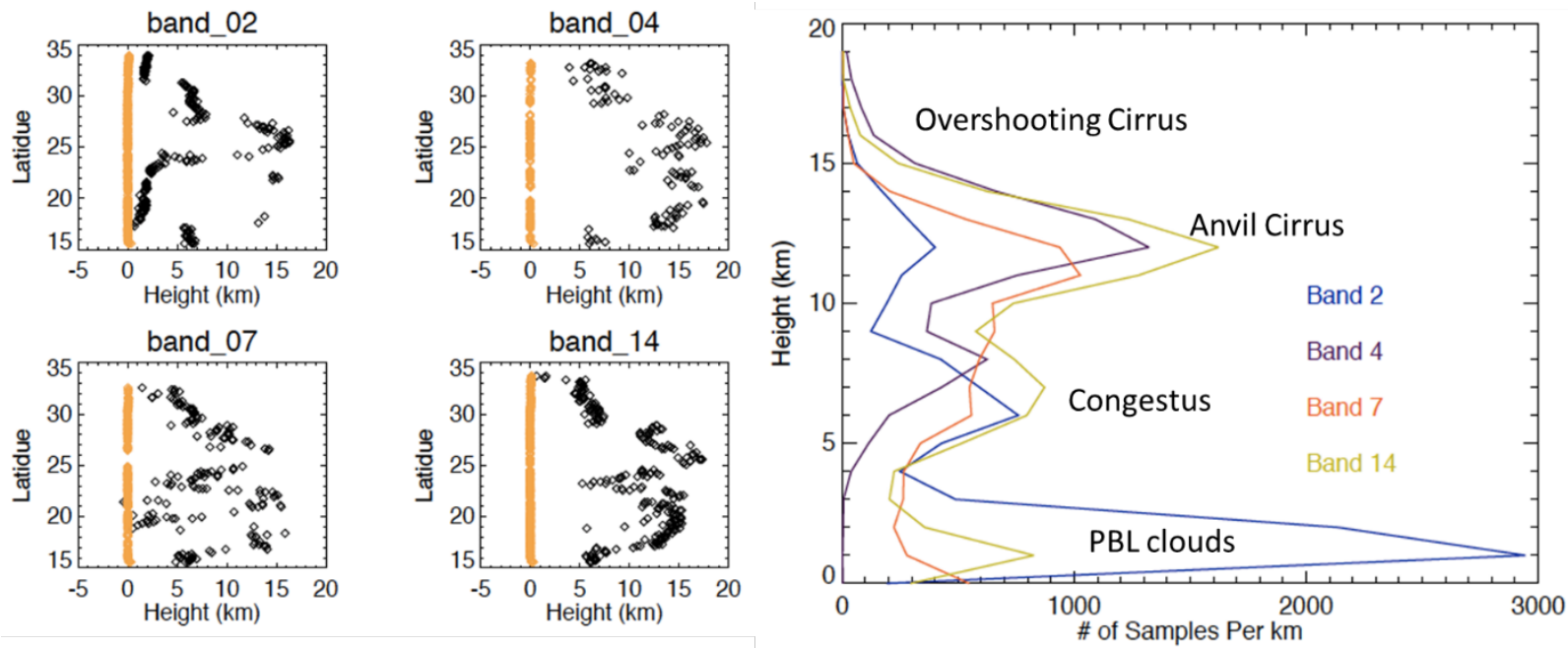

(a)

(b)

Figure 30. (a) Stereo height retrievals along $87.5^{\circ} \mathrm{W}$ longitude for the four spectral bands. The surface height is in orange. (b) Vertical distributions of the AMV stereo heights derived from the four spectral bands for the entire granule. All height retrievals are from chip size $32 \times 32 \mathrm{~km}$.

Figure 30b summarizes the vertical distribution of AMV height retrievals from the four spectral bands, all revealing a trimodal distribution except band 4 . The trimodal height distribution, a unique tropical cloud property, consists of PBL clouds, congestus, and cirrus outflows. As expected for its low-cloud screening, band 4 observes only the two high-level modes. The observation of the trimodal cloud distribution reflects one of the stereo technique strengths, compared to other passive remote sensing methods for cloud height assignment. Wu et al. [26] evaluated several passive techniques for cloud height measurements by comparing them against active sensor data, and found that only stereo technique was able to clearly detect the trimodal height distribution. In their study, the IR height-assignment method tended to often mix up low- and middle-level clouds with its height assignment, whereas the UV method suffers from the similar problem in addition to its poor sensitivity to high clouds.

Finally, retrieving stereo heights from IR images is particularly important for nighttime observations, despite a finer pixel resolution with the visible band. Both bands 7 and 14 are able to produce stereo heights in the absence of sunlight while band 7 may have a better sensitivity to low clouds over land (not shown). Among the thermal IR bands listed in Figure 29, our test results show that their coverage and sensitivity are all similar to band 14, but not significantly better than band 14 . 


\subsubsection{California Camp Fire (November 2018)}

Several California wildfires broke out in November 2018, including the Camp Fire in Northern California and the Woolsey Fire in the south, as revealed in the MODIS images on November 9-10 (Figures 31 and 32). In particular, the Camp Fire was one of the deadliest wildfires in California history, causing fatalities of 85 , property damage over $\$ 16 \mathrm{~B}$, and evacuation of 52,000 people in 13 counties. It started on November 8 th, and quickly spread to 20,000 acres on November 9th and 100,000 acres by November 10 th with only $20 \%$ containment. It lasted till November 25 th before it was $100 \%$ contained.

On November 9th, the fire entered the town of Paradise. Figure 31 captured a moment of rapid development of Camp Fire at 18:55 UTC (11:55 Local Time), showing $\sim 5 \mathrm{~m} / \mathrm{s}$ northwesterly winds at the top of the plume. The leading edge of plume, along the slice west-to-east, has a higher altitude than the trailing edge, decreasing steadily from $4 \mathrm{~km}$ to $2 \mathrm{~km}$. The elevated fire plume $(\sim 4 \mathrm{~km})$ suggests intense burning and heating on the ground. In the case of very intensified burning, strong fires can send a pyroculonimubus cloud up to $\sim 12 \mathrm{~km}$ [27]. In the Paradise case, wind speeds of $22 \mathrm{~m} / \mathrm{s}$ were reported at the ground on November 9th, allowing the fire to grow rapidly. Because of such rapid development, many residents in Paradise were unable to evacuate before the fire arrived, and firefighters were unable to prevent the flames from spreading.



Figure 31. (a) California camp fire and Woolsey fire as seen by Aqua/MODIS on November 9th. (b) The MODIS-GOES AMV and height retrievals over the northern California, and the cross-section (c) at a latitude of $39.6^{\circ} \mathrm{N}$. The AMV vectors in the map are colored by their height above terrain. In the $39.6^{\circ}$ $\mathrm{N}$ cross section, the terrain height is in orange whereas the 3D-wind stereo height is in black.

Figure 32 shows a scene of the northern California wildfires at 21:15 UTC on November 10th where Aqua/MODIS and GOES-16 made a joint stereo retrieval of the AMV and height from the fire plumes. At this time, the wildfire near Dos Rios was more than $50 \%$ contained, but the Camp fire continued to spread its damage. As seen in the MODIS image, the plumes from the Camp Fire were 
dense with heights topped at $1-1.5 \mathrm{~km}$ above the surface. The plumes near the fire sources are slightly higher than those in the downstream, with a northwesterly wind speed of $\sim 5 \mathrm{~m} / \mathrm{s}$. By combining the stereo height and wind measurements with MODIS fire intensity data, 3D-wind products can help to improve the wildfire emission and dispersion models used for public health and air quality prediction [28].

Over the coastal ocean, the height of plumes transported by the winds on previous days appears to decrease with distance from the coastline, as expected with aerosol scavenging processes (i.e., wet and dry deposition) [29]. Northern California and its coast region had little cloud coverage (except for smoke plumes) during November 8-10, and therefore the dry deposition was likely the dominant process in removing large particulate matter (PM) in the fire plume. The observed decrease of plume height is indicative of dry deposition of large PMs that determines the plume aerosol optical depth (AOD) and visibility/contrast in the visible bands.

Dense fire plumes on November 9-10 also severely impacted the visibility at San Francisco International Airport (SFO). Hundreds of flights scheduled to depart or land at SFO were either delayed or canceled on these days due to poor visibility and air quality conditions caused by the camp fire. On November 10th (Figure 32), the Bay Area is covered by smoke from the Camp Fire with a top of $\sim 1.2 \mathrm{~km}$ above the surface. This unhealthy air was mixed within the PBL, causing poor air quality and reduced visibility on these two days.

The stereo height retrieval can track the terrain height quite well in the regions where neither clouds nor smoke are present. These regions are usually associated with a near-zero wind speed. In the $37.8^{\circ} \mathrm{N}$ cross section in Figure 32, the terrain height rises sharply to $\sim 3000 \mathrm{~m}$ towards the Sierra Nevada Mountains. Although the 3D-wind algorithm does not retrieve every highly varying mountain peak with $8 \mathrm{~km}$ spatial resolution, it shows that the height retrievals can track smoothed mountain heights within $200 \mathrm{~m}$, which provides further validation on the quality of the stereo height retrieval.

(a)

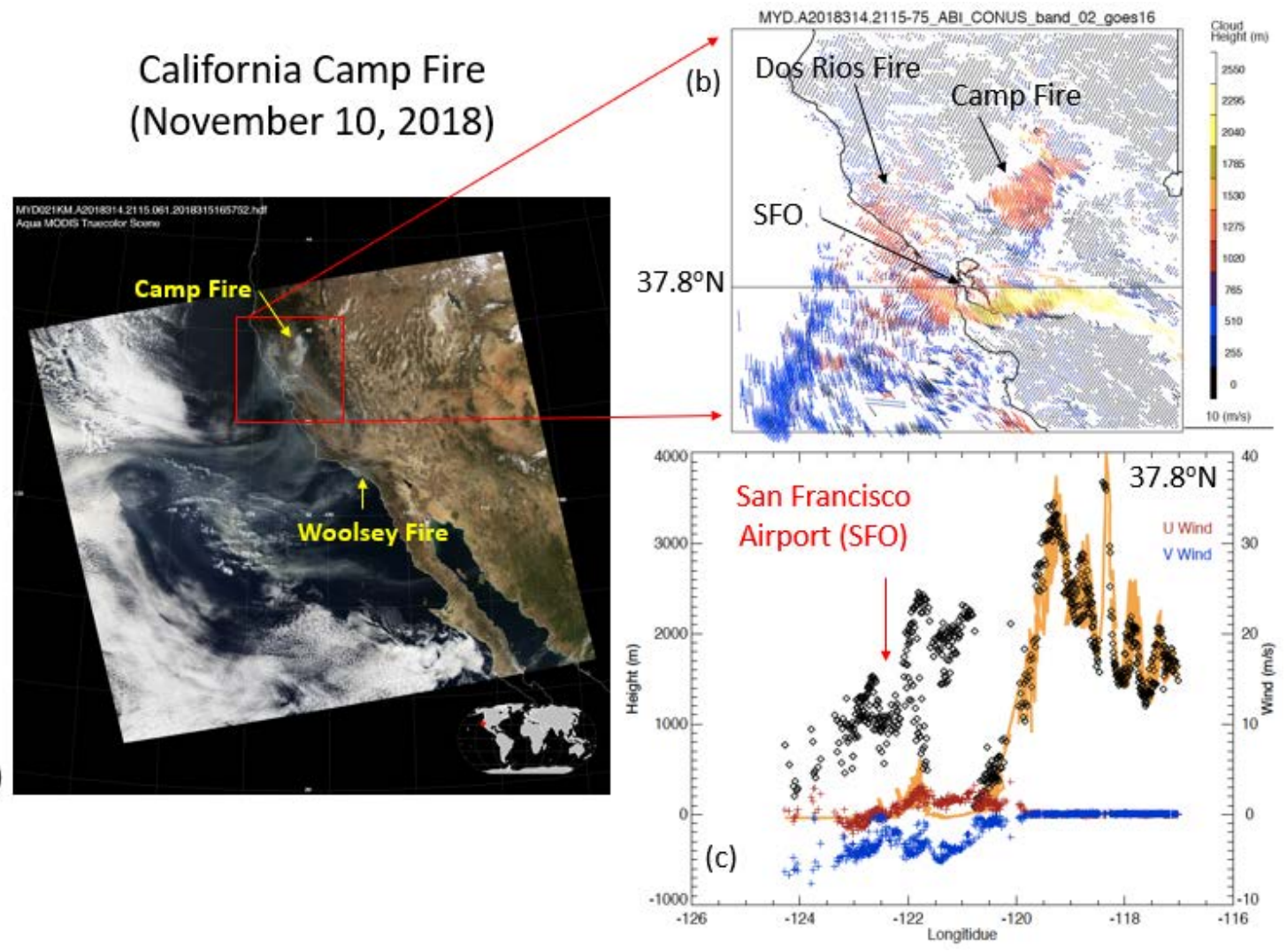

Figure 32. California camp fire and Woolsey fires (a) are shown as in Figure 31 but for November 10th. The cross-section (c) of the full set of retrievals $(\mathbf{b})$ is at the latitude of SFO $\left(37.8^{\circ} \mathrm{N}\right)$. 


\section{Conclusions}

Our work demonstrates that high-accuracy AMVs can be jointly retrieved from contemporaneous MODIS and GOES-R series ABI imagery along with the geometric height of each AMV using stereo methods. This is an extension of our prior work with MISR and GOES [9]. The advantages of using MODIS with GOES for this application are that MODIS offers a wider swath ( $2330 \mathrm{~km}$ versus $380 \mathrm{~km}$ ) and eight thermal IR bands that pair well with those of ABI. AMVs and heights are jointly retrieved from measurements of the disparities between features seen in one image and the matches to those features in the others. In general, disparities represent a mixture of motion (wind information) and parallax (height information). The 3D-winds retrieval model is identical to that used in our previous MISR-GOES work (without bundle adjustment) and is universally applicable to LEO-GEO, GEO-GEO, and LEO-LEO pairings. The model accounts for the non-simultaneity between observations by using pixel time-tagging models and therefore no synchronization is needed between the observations of heterogeneous platforms. Our methods are enabled by the very accurate geolocation provided by both MODIS and ABI. In each of their highest resolution visible channels, the verified mean plus three-times standard deviation geolocation error is $\sim 0.5$ pixels.

We have validated our methods by comparing MODIS-GOES 3D-wind retrievals against MISR-GOES retrievals and NOAA operational wind products, and through clear-sky terrain retrievals. The validation shows consistency in the retrieved winds between the various methods. We show a collection of clear-sky terrain retrievals using the highest resolution bands of MODIS and GOES that have small height biases $(<20 \mathrm{~m})$ and $\sim 120 \mathrm{~m}$ random errors. Its retrieved winds are essentially unbiased $(<0.03 \mathrm{~m} / \mathrm{s})$ and have $\sim 0.2 \mathrm{~m} / \mathrm{s}$ random errors in wind velocity. More cases would be of course necessary to establish accuracy bounds over a broader population of cases. The retrieval accuracy also depends on spectral band pairings and their spatial resolutions. Thermal-IR channels where the ABI resolution is $2 \mathrm{~km}$ instead of $0.5 \mathrm{~km}$ should be less accurate in general. It is also especially important to note that retrieval accuracy is sensitive to the relative geometry between MODIS and GOES. There is a singular geometry in which the lines of sight from each system to the feature are parallel and it is impossible to retrieve the AMV height in this situation. The retrieval uncertainty becomes infinite in the singular geometry and large in its neighborhood. The retrieval model provides a covariance estimate of the uncertainty of the retrieved model states (two velocities and three positions) that can be used to estimate jointly retrieved AMV and height uncertainties. Highly uncertain retrievals such as those near the singular geometry should be suppressed.

This new method offers many exciting possibilities for applications in atmospheric research and operational meteorology where accurate vertical resolution of atmospheric dynamics is important. The wide swath, many spectral channels, and multiplicity of MODIS and MODIS-like (e.g., VIIRS) sensors in orbit are greatly beneficial for the spatiotemporal coverage of 3D-winds enabled by our techniques. We showcased several examples of science applications, including probing the structure and dynamics of the PBL during a cold air utbreak over the Great Lakes of the United States, studying the vertically resolved dynamics of Hurricane Michael including deep convection using four spectral bands, and a study of wildfire smoke plumes. In all the cases, the horizontal and vertical resolutions provided by our methods are enabling of finely detailed descriptions of the phenomena under study. There is no doubt many more applications exist in addition to the examples that we have provided, and we welcome other investigators to explore them.

Author Contributions: Conceptualization, J.L.C., D.L.W.; methodology, J.L.C., D.L.W., R.E.W., G.L., B.T.; software, J.L.C., H.M., B.T.; validation, B.T., H.M., J.L.C.; formal analysis, J.L.C., D.L.W.; investigation, J.L.C., D.L.W.; resources, D.L.W., R.E.W.; data curation, J.L.C., H.M.; writing-original draft preparation, J.L.C., D.L.W., H.M., G.L., B.T., R.E.W.; writing-J.L.C., D.L.W., H.M., G.L., B.T., R.E.W.; visualization, J.L.C., D.L.W., H.M.; supervision, D.L.W., R.E.W.; project administration, D.L.W., R.E.W.; funding acquisition, D.L.W., R.E.W. 
Funding: Carr Astronautics was supported through a task on the Support for Atmospheres, Modeling, and Data Assimilation (SAMDA) contract at NASA Goddard Space Flight Center (NNG17HP01C), prime contractor, Science Systems and Applications, Inc. (SSAI). Gary Lin and Bin Tan were supported through a task on the Hydrospheric and Biospheric Science (HBS) Support Services contract at NASA Goddard Space Flight Center (NNG15HG01C), managed by Science Systems and Applications, Inc. (SSAI). The work of Dong Wu and Robert Wolfe was supported by NASA's Terra project.

Acknowledgments: Thanks to Marco Concha of the NASA GOES-R program for providing a mission-life ephemeris for both GOES-R satellites. Thanks also to Diego Principe, NASA/GSFC summer intern, for running several of the cases presented here. Special thanks to Stacey Williamson at Carr Astronautics who assisted with the bibliography and final production of the manuscript.

Conflicts of Interest: The authors declare no conflict of interest.

\section{References}

1. Menzel, W.P.; Smith, W.L.; Stewart, T.R. Improved cloud motion wind vector and altitude assignment using VAS. J. Clim. Appl. Meteorol. 1983, 22, 377-384. [CrossRef]

2. Roebeling, R.; Baum, B.; Bennartz, R.; Hamann, U.; Heidinger, A.; Thoss, A.; Walther, A. Evaluating and improving cloud parameter retrievals. Bull. Am. Meteorol. Soc. 2013, 94, ES41-ES44. [CrossRef]

3. Holz, R.E.; Ackerman, S.A.; Nagle, F.W.; Frey, R.; Dutcher, S.; Kuehn, R.E.; Vaughan, M.A.; Baum, B. Global Moderate Resolution Imaging Spectroradiometer (MODIS) cloud detection and height evaluation using CALIOP. J. Geophys. Res. Atmos. 2008, 113. [CrossRef]

4. Bedka, K.; Brunner, J.; Dworak, R.; Feltz, W.; Otkin, J.; Greenwald, T. Objective satellite-based detection of overshooting tops using infrared window channel brightness temperature gradients. J. Appl. Meteorol. Climatol. 2010, 49, 181-202. [CrossRef]

5. Nieman, S.J.; Schmetz, J.; Menzel, W.P. A comparison of several techniques to assign heights to cloud tracers. J. Appl. Meteorol. 1993, 32, 1559-1568. [CrossRef]

6. Chapel, J.; Stancliffe, D.; Bevacqua, T.; Winkler, S.; Clapp, B.; Rood, T.; Gaylor, D.; Freesland, D.; Krimchansky, A. Guidance, navigation, and control performance for the GOES-R spacecraft. CEAS Space J. 2015, 7, 87-104. [CrossRef]

7. Tan, B.; Dellomo, J.; Wolfe, R.; Reth, A. GOES-16 ABI navigation assessment. In Earth Observing Systems XXIII; SPIE: Bellingham, DC, USA, 2018; Volume 10764. [CrossRef]

8. Wolfe, R.E.; Nishihama, M.; Fleig, A.J.; Kuyper, J.A.; Roy, D.P.; Storey, J.C.; Patt, F.S. Achieving sub-pixel geolocation accuracy in support of MODIS land science. Remote Sens. Environ. 2002, 83, 31-49. [CrossRef]

9. Carr, J.L.; Wu, D.L.; Kelly, M.A.; Gong, J. MISR-GOES 3D Winds: Implications for future LEO-GEO and LEO-LEO winds. Remote Sens. 2018, 10, 1885. [CrossRef]

10. Carlomusto, M. GOES R Series Product Definition and Users' Guide (PUG): Volume 1; Main. 2018. Available online: https://www.goes-r.gov/users/docs/PUG-main-vol1.pdf (accessed on 20 July 2019).

11. MODIS Characterization Support Team. MODIS Level 1B Product User's Guide. Available online: https://mcst.gsfc.nasa.gov/sites/default/files/file_attachments/M1054E_PUG_2017_0901_V6.2.2_ Terra_V6.2.1_Aqua.pdf (accessed on 24 July 2019).

12. Carr, J.L. Georegistration of meteorological images. In Image Registration for Remote Sensing; Le Moigne, J., Netanyahu, N.S., Eastman, R.D., Eds.; Cambridge University Press: Cambridge, UK, 2011; pp. 339-354. [CrossRef]

13. Xiong, X.; Che, N.; Barnes, W. Terra MODIS on-orbit spatial characterization and performance. IEEE Trans. Geosci. Remote Sens. 2005, 43, 355-365. [CrossRef]

14. Xiong, X.; Che, N.; Barnes, W.; Xie, Y.; Wang, L.; Qu, J. Status of Aqua MODIS spatial characterization and performance. In Sensors, Systems, and Next-Generation Satellites X; SPIE: Bellingham, DC, USA, 2006; Volume 6361.

15. Xiong, X.; MODIS Characterization Support Team (MCST). MODIS Instrument Operations Status. In Proceedings of the October 2018 MODIS Science Team Meeting, Silver Spring, MD, USA, 15-19 October 2018; Available online: https://modis.gsfc.nasa.gov/sci_team/meetings/201810/calibration.php (accessed on 30 July 2019). 
16. Wolfe, R.E.; Nishihama, M. Accurate MODIS global geolocation through automated ground control image matching. In Image Registration for Remote Sensing; Le Moigne, J., Netanyahu, N.S., Eastman, R.D., Eds.; Cambridge University Press: Cambridge, UK, 2011; pp. 437-455.

17. Lin, G.; Wolfe, R.E.; Tilton, J.C.; Zhang, P.; Dellomo, J.J.; Tan, B. (Terra, Aqua) MODIS Geolocation Status. In Proceedings of the October 2018 MODIS Science Team Meeting, Silver Spring, MD, USA, 15-19 October 2018. Available online: https://modis.gsfc.nasa.gov/sci_team/meetings/201810/calibration.php (accessed on 4 September 2019).

18. Cao, C.; Luccia, F.J.D.; Xiong, X.; Wolfe, R.; Weng, F. Early on-orbit performance of the visible infrared imaging radiometer suite onboard the Suomi National Polar-Orbiting Partnership (S-NPP) satellite. IEEE Trans. Geosci. Remote Sens. 2014, 52, 1142-1156. [CrossRef]

19. Wolfe, R.E.; Lin, G.; Nishihama, M.; Tewari, K.P.; Tilton, J.C.; Isaacman, A.R. Suomi NPP VIIRS prelaunch and on-orbit geometric calibration and characterization. J. Geophys. Res. Atmos. 2013, 118, 11508-11521. [CrossRef]

20. Lin, G.; Wolfe, R.E.; Tilton, J.C.; Zhang, P.; Dellomo, J.J.; Tan, B. JPSS-1/NOAA-20 VIIRS early on-orbit geometric performance. In Earth Observing Systems XXIII; SPIE: Bellingham, DC, USA, 2018; Volume 10764.

21. Moroney, C.; Horvath, A.; Davies, R. Use of stereo-matching to coregister multiangle data from MISR. IEEE Trans. Geosci. Remote Sens. 2002, 40, 1541-1546. [CrossRef]

22. Young, G.S.; Kristovich, D.A.; Hjelmfelt, M.R.; Foster, R.C. Rolls, streets, waves, and more: A review of quasi-two-dimensional structures in the atmospheric boundary layer. Bull. Am. Meteorol. Soc. 2002, 83, 997-1002. [CrossRef]

23. Brümmer, B.; Pohlmann, S. Wintertime roll and cell convection over Greenland and Barents Sea regions: A climatology. J. Geophys. Res. Atmos. 2000, 105, 15559-15566. [CrossRef]

24. Garratt, J.R. The internal boundary layer-A review. Bound. Layer Meteorol. 1990, 50, 171-203. [CrossRef]

25. Wu, D.L.; Lee, J.N. Arctic low cloud changes as observed by MISR and CALIOP: Implication for the enhanced autumnal warming and sea ice loss. J. Geophys. Res. Atmos. 2012, 117. [CrossRef]

26. Wu, D.L.; Ackerman, S.A.; Davies, R.; Diner, D.J.; Garay, M.J.; Kahn, B.H.; Maddux, B.C.; Moroney, C.M.; Stephens, G.L.; Veefkind, J.P.; et al. Vertical distributions and relationships of cloud occurrence frequency as observed by MISR, AIRS, MODIS, OMI, CALIPSO, and CloudSat. Geophys. Res. Lett. 2009, 36. [CrossRef]

27. Fromm, M.; Lindsey, D.T.; Servranckx, R.; Yue, G.; Trickl, T.; Sica, R.; Doucet, P.; Godin-Beekmann, S. The untold story of pyrocumulonimbus. Bull. Am. Meteorol. Soc. 2010, 91, 1193-1210. [CrossRef]

28. Goodrick, S.L.; Achtemeier, G.L.; Larkin, N.K.; Liu, Y.; Strand, T.M. Modelling smoke transport from wildland fires: A review. Int. J. Wildland Fire 2013, 22, 83-94. [CrossRef]

29. Urbanski, S.P.; Hao, W.M.; Baker, S. Chapter 4 chemical composition of wildland fire emissions. In Developments in Environmental Science; Bytnerowicz, A., Arbaugh, M.J., Riebau, A.R., Andersen, C., Eds.; Elsevier: Amsterdam, The Netherlands, 2008; Volume 8, pp. 79-107. 